\title{
Deriving the mass of particles from Extended Theories of Gravity in LHC era
}

\author{
Salvatore Capozziello \\ Dipartimento di Scienze Fisiche, Università di Napoli "Federico II" and INFN Sez. di Napoli, \\ Compl. Univ. di Monte S. Angelo, Edificio G, Via Cinthia, I-80126, Napoli, Italy, \\ Giuseppe Basini \\ CERN, CH-1211, Geneva 23, Switzerland and Laboratori Nazionali di Frascati, \\ INFN, Via E. Fermi, C.P. 13, I-0044 Frascati, Italy, \\ Mariafelicia De Laurentis \\ Dip. di Scienze Fisiche, Università di Napoli "Federico II" and INFN Sez. di Napoli, \\ Compl. Univ. di Monte S. Angelo, Edificio G, Via Cinthia, I-80126, Napoli, Italy.
}

(Dated: December 2, 2018)

\begin{abstract}
We derive a geometrical approach to produce the mass of particles that could be suitably tested at LHC. Starting from a 5D unification scheme, we show that all the known interactions could be suitably deduced as an induced symmetry breaking of the non-unitary $G L(4)$-group of diffeomorphisms. The deformations inducing such a breaking act as vector bosons that, depending on the gravitational mass states, can assume the role of interaction bosons like gluons, electroweak bosons or photon. The further gravitational degrees of freedom, emerging from the reduction mechanism in $4 \mathrm{D}$, eliminate the hierarchy problem since generate a cut-off comparable with electroweak one at $\mathrm{TeV}$ scales. In this "economic" scheme, gravity should induce the other interactions in a non-perturbative way.

Keywords: Gravitational field; unified theories; group theory; fiber bundle formalism; mass of particles.
\end{abstract}

\section{INTRODUCTION}

The Standard Model of Particles can be considered a successful relativistic quantum field theory both from particle physics and group theory points of view. Technically, it is a non-Abelian gauge theory (a Yang-Mills theory) associated with the tensor product of the internal symmetry groups $S U(3) \times S U(2) \times U(1)$, where the $S U(3)$ color symmetry for quantum chromodynamics, is treated as exact, whereas the $S U(2) \times U(1)$ symmetry, responsible for generating the electro-weak gauge fields, is considered spontaneously broken.

So far, as we know, there are four fundamental forces in Nature; namely, electromagnetic, weak, strong and gravitational forces. The Standard Model well represents the first three, but not the gravitational interaction. On the other hand, General Relativity (GR) is a geometric theory of the gravitational field which is described by the metric tensor $g_{\mu \nu}$ defined on pseudo-Riemannian spacetimes. The Einstein field equations are nonlinear and have to be satisfied by the metric tensor. This nonlinearity is indeed a source of difficulty in quantization of General Relativity. Since the Standard Model is a gauge theory where all the fields mediating the interactions are represented by gauge potentials, the question is why the fields mediating the gravitational interaction are different from those of the other fundamental forces. It is reasonable to expect that there may be a gauge theory in which the gravitational fields stand on the same footing as those of other fields 1]. As it is well-known, this expectation has prompted a re-examination of GR from the point of view of gauge theories.
While the gauge groups involved in the Standard Model are all internal symmetry groups, the gauge groups in GR must be associated with external space-time symmetries. Therefore, the gauge theory of gravity cannot be dealt under the standard of the usual Yang-Mills theories. It must be one in which gauge objects are not only gauge potentials but also tetrads that relate the symmetry group to the external space-time. For this reason we have to consider a more complex nonlinear gauge theory where all the interactions should be dealt under the same standard [3]. In GR, Einstein took the space-time metric components as the basic set of variables representing gravity, whereas Ashtekar and collaborators employed the tetrad fields and the connection forms as the fundamental variables [2]. We also will consider the tetrads and the connection forms as the fundamental fields but with the difference that this approach gives rise to a covariant symplectic formalism capable of achieving the result of dealing with physical fields under the same standard [4, 5].

In order to frame historically our approach, let us sketch a quick summary of the various attempts where the Standard Model and GR have been considered under the same comprehensive picture. In 1956, Utiyama suggested that gravitation may be viewed as a gauge theory [6] in analogy to the Yang-Mills [7] theory (1954). He identified the gauge potential due to the Lorentz group with the symmetric connection of the Riemann geometry, and reproduced the Einstein GR as a gauge theory of the Lorentz group $S O(3,1)$ with the help of tetrad fields introduced in an ad hoc manner. Although the tetrads were necessary components of the theory (to relate the Lorentz group) adopted as an internal gauge group to the 
external space-time, they were not introduced as gauge fields. In 1961, Kibble 8] constructed a gauge theory based on the Poincaré group $P(3,1)=T(3,1) \rtimes S O(3,1)$ (the symbol $\rtimes$ represents the semi-direct product) which resulted in the Einstein-Cartan theory characterized by curvature and torsion. The translation group $T(3,1)$ is considered responsible for generating the tetrads as gauge fields. Cartan [9] generalized the Riemann geometry in order to include torsion in addition to curvature. The torsion (tensor) arises from an asymmetric connection. Sciama [10], and others (Fikelstein [11], Hehl [12, 13]) pointed out that intrinsic spin may be the source of torsion of the underlying space-time manifold.

Since the form and the role of tetrad fields are very different from those of gauge potentials, it has been thought that even Kibble's attempt is not satisfactory as a full gauge theory. There have been a number of gauge theories of gravitation based on a variety of Lie groups [12, 13, 15 19]. It was argued that a gauge theory of gravitation corresponding to GR can be constructed with the translation group alone, in the so-called teleparallel scheme [14].

Inomata et al. [20] proposed that Kibble's gauge theory could be obtained, in a way closer to the Yang-Mills approach, by considering the de Sitter group $S O(4,1)$, which is reducible to the Poincaré group by a groupcontraction. Unlike the Poincaré group, the de Sitter group is homogeneous and the associated gauge fields are all of gauge potential type and by the Wigner-Inönu group contraction procedure, one of the five vector potentials reduces to the tetrad.

It is standard to use the fiber-bundle formulation by which gauge theories can be constructed on the basis of any Lie group. Works by Hehl et al. [19], on the so-called Metric Affine Gravity (MAG), adopted as a gauge group the affine group $A(4, \mathbb{R})=T(4) \rtimes G L(4, \mathbb{R})$, which can be linearly realized. The tetrad has been identified by the nonlinearly realized translational part of the affine connection, on the tangent bundle. In MAG theory, the Lagrangian is quadratic in both curvature and torsion, in contrast to the Einstein-Hilbert Lagrangian of GR which is linear in the scalar curvature. The theory has the Einstein limit on one hand and leads to the Newtonian inverse distance potential plus the linear confinement potential (in the weak field approximation) on the other hand. In summary, as we have seen, there are many attempts to formulate gravitation as a gauge theory but currently no theory has been uniquely accepted as the gauge theory of gravity.

The nonlinear approach to group realizations was originally introduced by Coleman, Wess and Zumino [21, 22] in the context of internal symmetry groups (1969). It was later extended to the case of space-time symmetries by Isham, Salam, and Strathdee [23] considering the nonlinear action of $G L(4, \mathbb{R})$, modulus the Lorentz subgroup. In 1974, Borisov, Ivanov and Ogievetsky [24, 25], considered the simultaneous nonlinear realization (NLR) of the affine and conformal groups. They stated that GR can be viewed as a consequence of spontaneous breakdown of the affine symmetry, in the same way that chiral dynamics, in quantum chromodynamics, is a result of spontaneous breakdown of chiral symmetry. In their model, gravitons are considered as Goldstone bosons associated with the affine symmetry breaking. As we will see below, this approach can be pursued in general.

In 1978, Chang and Mansouri 26] used the NLR scheme adopting $G L(4, \mathbb{R})$ as the principal group. In 1980, Stelle and West 27] investigated the NLR induced by the spontaneous breakdown of $S O(3,2)$. In 1982 Ivanov and Niederle considered nonlinear gauge theories of the Poincaré, de Sitter, conformal and special conformal groups [28, 29]. In 1983, Ivanenko and Sardanashvily [30] considered gravity to be a spontaneously broken $G L(4, \mathbb{R})$ gauge theory. The tetrads fields arise, in their formulation, as a result of the reduction of the structure group of the tangent bundle from the general linear to Lorentz group. In 1987, Lord and Goswami [31, 32] developed the NLR in the fiber bundle formalism based on the bundle structure $G(G / H, H)$ as suggested by Ne'eman and Regge 33. In this approach, the quotient space $G / H$ is identified with physical spacetime. Most recently, in a series of papers, Lopez-Pinto, Julve, Tiemblo, Tresguerres and Mielke discussed nonlinear gauge theories of gravity on the basis of the Poincaré, affine and conformal groups $[34,39]$.

Now, following the prescriptions of GR, the physical space-time is assumed to be a four-dimensional differential manifold. In Special Relativity (SR), this manifold is the Minkwoski flat-space-time $M_{4}$ while, in GR, the underlying space-time is assumed to be curved in order to describe the effects of gravitation.

As we said, Utiyama [6] proposed that GR can be seen as a gauge theory based on the local Lorentz group in the same way that the Yang-Mills gauge theory [7] is developed on the basis of the internal iso-spin gauge group. In this formulation, the Riemannian connection is the gravitational counterpart of the Yang-Mills gauge fields. While $S U(2)$, in the Yang-Mills theory, is an internal symmetry group, the Lorentz symmetry represents the local nature of space-time rather than internal degrees of freedom. The Einstein Equivalence Principle, asserted for GR, requires that the local space-time structure can be identified with the Minkowski space-time possessing Lorentz symmetry.

In order to relate local Lorentz symmetry to the external space-time, we need to solder the local space to the external space. The soldering tools can be the tetrad fields. Utiyama regarded the tetrads as objects given $a$ priori while they can be dynamically generated [3] and the space-time has necessarily to be endowed with torsion in order to accommodate spinor fields [40]. In other words, the gravitational interaction of spinning particles requires the modification of the Riemann space-time of GR to be a (non-Riemannian) curved space-time with torsion. Although Sciama used the tetrad formalism for his gauge-like handling of gravitation, his theory fell 
shortcomings in treating tetrad fields as gauge fields.

Following the Kibble approach [8], it can be demonstrated how gravitation can be formulated starting from a pure gauge viewpoint. In particular, gravity can be seen as a gauge theory which can be obtained starting from some local invariance, e.g. the local Poincaré symmetry, leading to a suitable unification scheme [3]. This dynamical structure can be based on a nonlinear realization of the local conformal-affine group of symmetry transformations [41].

Here, we start from a General Invariance Principle, as requested in the so called Open Quantum Relativity (OQR) [1, 42] and consider first the Global Poincaré Invariance and then the Local Poincaré Invariance. This approach leads to construct a given theory of gravity as a gauge theory. Such a viewpoint, if considered in detail, can avoid many shortcomings and could be useful to formulate self-consistent schemes for quantum gravity and then the unification of all interactions [3].

In particular, the idea of an unification theory, capable of describing all the fundamental interactions of physics under the same standard, has been one of the main issues of modern physics, starting from the early efforts of Einstein, Weyl, Kaluza and Klein [43] until the most recent approaches [44]. Nevertheless, the large number of ideas, up to now proposed, which we classify as unified theories, results unsuccessful due to several reasons: the technical difficulties connected with the lack of a unitary mathematical description of all the interactions; the huge number of parameters introduced to "build up" the unified theory and the fact that most of them cannot be observed neither at laboratory nor at astrophysical (or cosmological) conditions [45]; the very wide (and several times questionable since not-testable) number of extradimensions requested by several approaches. Due to this situation, it seems that unification is a useful (and aesthetic) paradigm, but far to be achieved, if the trend is continuing to try to unify interactions (i.e. to make something simple) by adding and adding ingredients: new particles and new parameters (e.g. dark matter forest). A different approach could be to consider the very essential physical quantities and try to achieve unification without any ad hoc new ingredients. This approach can be pursued starting from straightforward considerations which lead to reconsider modern physics under a sort of economic issue: let us try to unifying forces approaching new schemes but without adding new parameters 1 . A prominent role deserves the conservation laws and the fact that each of them brings out the existence of a symmetry [3].

As a general remark, the Noether Theorem states that, for every conservation law of Nature, a symmetry must exist. This leads to a fundamental result also from a

\footnotetext{
${ }^{1}$ Following Occam's Razor prescription: Entia non sunt multiplicanda praeter necessitatem.
}

mathematical point of view since the presence of symmetries technically reduces dynamics (i.e. gives rise to first integrals of motion) and, in several cases, allows to get the general solution. With these considerations in mind, we can try to change our point of view and investigate what will be the consequences of the absolute validity of conservation laws without introducing any arbitrary symmetry breaking.

In order to see what happens as soon as we ask for the absolute validity of conservation laws, we could take into account the Bianchi identities. Such geometrical identities work in every covariant field theory (e.g. Electromagnetism or GR) and can be read as equations of motion also in a fiber bundle approach [106]. We want to show that, the absolute validity of conservation laws, intrinsically contains symmetric dynamics; moreover, reducing dynamics from $5 \mathrm{D}$ to $4 \mathrm{D}$, it gives rise to the physical quantities characterizing particles as the mass.

The minimal ingredient which we require to achieve these results is the fact that a 5-dimensional, singularity free space, where conservation laws are always and absolutely conserved, has to be defined. Specifically, in such a space, Bianchi identities are asked to be always valid and, moreover, the process of reduction to $4 \mathrm{D}$-space generates the mass spectra of particles. In this sense, a dynamical unification scheme will be achieved where a fifth dimension has the physical meaning of inducing the mass of particles by deformations of space-time. In other words, we will show that deformations can be parameterized as "effective" scalar fields in a $G L(4)$-group of diffeomorphisms. In this sense, we do not need any spontaneous symmetry breaking but just a self-consistent way to classify deformations as "gauge bosons". The layout of the paper is the following. In Sec.II, we discuss in detail the conformal-affine structure of gravitational field showing that the nonlinear realization of a group provides a way to determine the transformation properties of fields defined on a given quotient space $G / H$. In other words, we show that gravitational field can be realized in many equivalent ways and we will use this feature to show that gravitational massive states are possible. Sec. III is devoted to the group structure. We show that the 4D-group of diffeomorphisms can be embedded in that in 5D. Furthermore, it is straightforward to show that $G L(4)$ contains all the generators of the Standard Model plus generators of the gravitational field. The space-time deformations as elements of $G L(4)$-group are discussed in Sec.IV. The main result of this section is that deformations can be dealt as effective geometric scalar fields. In Sec.V, the 5D space-time structure and the reduction to 4 D-dynamics is discussed. Such a reduction mechanism gives rise to effective theories of gravity (Extended Theories of Gravity [46 50]) where higher-order terms in curvature invariants or nonminimal couplings are naturally achieved. In Sec.VI, we discuss that these effective theories can be conformally related and the only singular theory (with null Hessian determinant) is GR. The straightforward consequence of such a result is that gravi- 
tational massive modes can be always generated. Sec.VII is devoted to the discussion of the mass generation while, in Sec.VIII, we derive massless and massive gravitational modes related to Extended Theories of Gravity. An interesting byproduct is the fact that 6 polarization states emerge and this result is perfectly in agreement with the fundamental Riemann theorem stating that in a $N$ dimensional space, $N(N-1) / 2$ gravitational degrees of freedom are allowed. Sec.IX is devoted to the specific issue that massive gravitons could have observable effects between $\mathrm{GeV}-\mathrm{TeV}$ scales and induce a symmetry breaking through a sort of regularization mechanism. Conclusions are drawn in Sec.X.

\section{THE CONFORMAL-AFFINE STRUCTURE OF GRAVITATIONAL FIELD}

\section{A. Generalities on fiber bundle formalism}

In this section, we shall take into account the fiber bundle formalism of gravitational field showing that it naturally exhibit a conformal-affine structure. This feature, in some sense, allow to compare all the theories of gravity, based on diffeomorphism invariance, under the same standard.

Before considering in details the conformal-affine structure of gravitational theories, let us briefly review the standard bundle approach to gauge theories. First, let us show that a usual gauge potential $\Omega$ is the pullback of 1 -form connection $\omega$ by the local sections of the bundle. Then, the transformation laws of the $\omega$ and $\Omega$ under the action of the structure group $G$ are deduced.

Modern formulations of gauge field theories are geometrically expressible in the language of principal fiber bundles. A fiber bundle is a structure $\langle\mathbb{P}, M, \pi ; \mathbb{F}\rangle$ where $\mathbb{P}$ (the total bundle space) and $M$ (the base space) are smooth manifolds, $\mathbb{F}$ is the fiber space and the surjection $\pi$ (a canonical projection) is a smooth map of $\mathbb{P}$ onto $M$,

$$
\pi: \mathbb{P} \rightarrow M .
$$

The inverse image $\pi^{-1}$ is diffeomorphic to $\mathbb{F}$

$$
\pi^{-1}(x) \equiv \mathbb{F}_{x} \approx \mathbb{F},
$$

and it is called the fiber at $x \in M$. The partitioning $\bigcup_{x} \pi^{-1}(x)=\mathbb{P}$ is referred to as the fibration. Note that a smooth map is one whose coordinatization is $C^{\infty}$ differentiable; a smooth manifold is a space that can be covered with coordinate patches in such a manner that, a change from one patch to any overlapping patch is smooth [105]. Fiber bundles that admit a decomposition as a direct product, locally looking like $\mathbb{P} \approx M \times \mathbb{F}$, are called trivial. Given a set of open coverings $\left\{\mathcal{U}_{i}\right\}$ of $M$ with $x \in\left\{\mathcal{U}_{i}\right\} \subset M$ satisfying $\bigcup_{\alpha} \mathcal{U}_{\alpha}=M$, the diffeomorphism map is given by

$$
\chi_{i}: \mathcal{U}_{i} \times{ }_{M} G \rightarrow \pi^{-1}\left(\mathcal{U}_{i}\right) \in \mathbb{P},
$$

$\left(\times_{M}\right.$ represents the fiber product of elements defined over the space $M)$ such that $\pi\left(\chi_{i}(x, g)\right)=x$ and $\chi_{i}(x, g)=\chi_{i}\left(x,(i d)_{G}\right) g=\chi_{i}(x) g \forall x \in\left\{\mathcal{U}_{i}\right\}$ and $g \in G$. Here, $(i d)_{G}$ represents the identity element of the group $G$. In order to obtain the global bundle structure, the local charts $\chi_{i}$ must be glued together continuously. Consider two patches $\mathcal{U}_{n}$ and $\mathcal{U}_{m}$ with a non-empty intersection $\mathcal{U}_{n} \cap \mathcal{U}_{m} \neq \emptyset$. Let $\rho_{n m}$ be the restriction of $\chi_{n}^{-1}$ to $\pi^{-1}\left(\mathcal{U}_{n} \cap \mathcal{U}_{m}\right)$ defined by $\rho_{n m}: \pi^{-1}\left(\mathcal{U}_{n} \cap \mathcal{U}_{m}\right) \rightarrow\left(\mathcal{U}_{n} \cap \mathcal{U}_{m}\right) \times_{M} G_{n}$. Similarly let $\rho_{m n}: \pi^{-1}\left(\mathcal{U}_{m} \cap \mathcal{U}_{n}\right) \rightarrow\left(\mathcal{U}_{m} \cap \mathcal{U}_{n}\right) \times_{M} G_{m}$ be the restriction of $\chi_{m}^{-1}$ to $\pi^{-1}\left(\mathcal{U}_{n} \cap \mathcal{U}_{m}\right)$. The composite diffeomorphism $\Lambda_{n m} \in G$

$$
\Lambda_{m n}:\left(\mathcal{U}_{n} \cap \mathcal{U}_{m}\right) \times G_{n} \rightarrow\left(\mathcal{U}_{m} \cap \mathcal{U}_{n}\right) \times_{M} G_{m},
$$

defined as

$$
\Lambda_{i j}(x) \equiv \rho_{j i} \circ \rho_{i j}^{-1}=\chi_{i, x} \circ \chi_{j, x}^{-1}: \mathbb{F} \rightarrow \mathbb{F},
$$

constitutes the transition function between bundle charts $\rho_{n m}$ and $\rho_{m n}$ (o represents the group composition operation) where the diffeomorphism $\chi_{i, x}: \mathbb{F} \rightarrow \mathbb{F}_{x}$ is written as $\chi_{i, x}(g):=\chi_{i}(x, g)$ and satisfies $\chi_{j}(x, g)=$ $\chi_{i}\left(x, \Lambda_{i j}(x) g\right)$. The transition functions $\left\{\Lambda_{i j}\right\}$ can be interpreted as passive gauge transformations. They satisfy some consistency conditions, i.e. the identity $\Lambda_{i i}(x)$, the inverse $\Lambda_{i j}(x)=\Lambda_{j i}^{-1}(x)$ and the cocycle $\Lambda_{i j}(x) \Lambda_{j k}(x)=\Lambda_{i k}(x)$. For trivial bundles, the transition function reduces to

$$
\Lambda_{i j}(x)=g_{i}^{-1} g_{j},
$$

where $g_{i}: \mathbb{F} \rightarrow \mathbb{F}$ is defined by $g_{i}:=\chi_{i, x}^{-1} \circ \tilde{\chi}_{i, x}$, provided the local trivializations $\left\{\chi_{i}\right\}$ and $\left\{\tilde{\chi}_{i}\right\}$ it gives rise to the same fiber bundle.

A section is defined as a smooth map

$$
s: M \rightarrow \mathbb{P},
$$

such that $s(x) \in \pi^{-1}(x)=\mathbb{F}_{x} \forall x \in M$ and satisfies

$$
\pi \circ s=(i d)_{M},
$$

where $(i d)_{M}$ is the identity element of $M$. It assigns to each point $x \in M$ a point in the fiber over $x$. Trivial bundles admit global sections.

A bundle is a principal fiber bundle $\langle\mathbb{P}, \mathbb{P} / G, G, \pi\rangle$ provided that the Lie group $G$ acts freely (i.e. if $p g=p$ then $\left.g=(i d)_{G}\right)$ on $\mathbb{P}$ to the right $R_{g} p=p g, p \in \mathbb{P}$, preserves fibers on $\mathbb{P}\left(R_{g}: \mathbb{P} \rightarrow \mathbb{P}\right)$, and finally is transitive on fibers. Furthermore, there must exist local trivializations compatible with the $G$ action. Hence, $\pi^{-1}\left(\mathcal{U}_{i}\right)$ is homeomorphic to $\mathcal{U}_{i} \times_{M} G$ and the fibers of $\mathbb{P}$ are diffeomorphic to $G$. The trivialization or inverse diffeomorphism map is given by

$$
\chi_{i}^{-1}: \pi^{-1}\left(\mathcal{U}_{i}\right) \rightarrow \mathcal{U}_{i} \times_{M} G
$$

such that $\chi^{-1}(p)=(\pi(p), \phi(p)) \in \mathcal{U}_{i} \times_{M} G, p \in$ $\pi^{-1}\left(\mathcal{U}_{i}\right) \subset \mathbb{P}$, where we see from the above definition 
that $\phi$ is a local mapping of $\pi^{-1}\left(\mathcal{U}_{i}\right)$ into $G$ satisfying $\phi\left(L_{g} p\right)=\phi(p) g$ for any $p \in \pi^{-1}(\mathcal{U})$ and any $g \in G$. Let us observe that the elements of $\mathbb{P}$ which are projected onto the same $x \in\left\{\mathcal{U}_{i}\right\}$ are transformed into one another by the elements of $G$. In other words, the fibers of $\mathbb{P}$ are the orbits of $G$ and at the same time, they are the set of elements which are projected onto the same $x \in \mathcal{U} \subset M$. This observation motivates calling the action of the group vertical and the base manifold horizontal. The diffeomorphism map $\chi_{i}$ is called the local gauge since $\chi_{i}^{-1}$ maps $\pi^{-1}\left(\mathcal{U}_{i}\right)$ onto the direct (Cartesian) product $\mathcal{U}_{i} \times_{M} G$. The action $L_{g}$ of the structure group $G$ on $\mathbb{P}$ defines an isomorphism of the Lie algebra $\mathfrak{g}$ of $G$ onto the Lie algebra of vertical vector fields on $\mathbb{P}$, tangent to the fiber at each $p \in \mathbb{P}$ called fundamental vector fields

$$
\lambda_{g}: T_{p}(\mathbb{P}) \rightarrow T_{g p}(\mathbb{P})=T_{\pi(p)}(\mathbb{P})
$$

where $T_{p}(\mathbb{P})$ is the space of tangents at $p$, i.e. $T_{p}(\mathbb{P}) \in$ $T(\mathbb{P})$. The map $\lambda$ is a linear isomorphism for every $p \in \mathbb{P}$ and is invariant with respect to the action of $G$, that is, $\lambda_{g}:\left(\lambda_{g *} T_{p}(\mathbb{P})\right) \rightarrow T_{g p}(\mathbb{P})$, where $\lambda_{g *}$ is the differential push forward map induced by $\lambda_{g}$ defined by $\lambda_{g *}: T_{p}(\mathbb{P}) \rightarrow T_{g p}(\mathbb{P})$.

Since the principal bundle $\mathbb{P}(M, G)$ is a differentiable manifold, we can define tangent $T(\mathbb{P})$ and cotangent $T^{*}(\mathbb{P})$ bundles. The tangent space $T_{p}(\mathbb{P})$ defined at each point $p \in \mathbb{P}$ may be decomposed into a vertical $V_{p}(\mathbb{P})$ and horizontal $H_{p}(\mathbb{P})$ subspace as $T_{p}(\mathbb{P}):=V_{p}(\mathbb{P}) \oplus H_{p}(\mathbb{P})$ (where $\oplus$ represents the direct sum). The space $V_{p}(\mathbb{P})$ is a subspace of $T_{p}(\mathbb{P})$ consisting of all tangent vectors to the fiber passing through $p \in \mathbb{P}$, and $H_{p}(\mathbb{P})$ is the subspace complementary to $V_{p}(\mathbb{P})$ at $p$. The vertical subspace $V_{p}(\mathbb{P}):=\left\{X \in T(\mathbb{P}) \mid \pi(X) \in \mathcal{U}_{i} \subset M\right\}$ is uniquely determined by the structure of $\mathbb{P}$, whereas the horizontal subspace $H_{p}(\mathbb{P})$ cannot be uniquely specified. This result is very important because it makes possible to fix the Cauchy conditions on the dynamics. Thus we require the following condition: when $p$ transforms as $p \rightarrow p^{\prime}=p g$, $H_{p}(\mathbb{P})$ transforms as [53],

$$
R_{g *} H_{p}(\mathbb{P}) \rightarrow H_{p^{\prime}}(\mathbb{P})=R_{g} H_{p}(\mathbb{P})=H_{p g}(\mathbb{P}) .
$$

Let the local coordinates of $\mathbb{P}(M, G)$ be $p=(x, g)$ where $x \in M$ and $g \in G$. Let $\mathbf{G}_{A}$ denote the generators of the Lie algebra $\mathfrak{g}$ corresponding to group $G$ satisfying the commutators $\left[\mathbf{G}_{A}, \mathbf{G}_{B}\right]=f_{A B}{ }^{C} \mathbf{G}_{C}$, where $f_{A B}{ }_{C}$ are the structure constants of $G$. Let $\Omega$ be a connection form defined by $\Omega^{A}:=\Omega_{i}^{A} d x^{i} \in \mathfrak{g}$. Let $\omega$ be a connection 1-form defined by

$$
\omega:=\widetilde{g}^{-1} \pi_{\mathbb{P} M}^{*} \Omega \widetilde{g}+\widetilde{g}^{-1} d \widetilde{g},
$$

(* represents the differential pullback map) belonging to $\mathfrak{g} \otimes T_{p}^{*}(\mathbb{P})$ where $T_{p}^{*}(\mathbb{P})$ is the dual space to $T_{p}(\mathbb{P})$. In such a case, the differential pullback map, applied to a test function $\phi$ and $p$-forms $\alpha$ and $\beta$, satisfies $f^{*} \phi=$ $\phi \circ f,(g \circ f)^{*}=f^{*} g^{*}$ and $f^{*}(\alpha \wedge \beta)=f^{*} \alpha \wedge f^{*} \beta$. If $G$ is represented by a $d$-dimensional $d \times d$ matrix, then
$\mathbf{G}_{A}=\left[\mathbf{G}_{\alpha \beta}\right], \widetilde{g}=\left[\widetilde{g}^{\alpha \beta}\right]$, where $\alpha, \beta=1,2,3, \ldots d$. Thus, $\omega$ assumes the form

$$
\omega_{\alpha}^{\beta}=\left(\widetilde{g}^{-1}\right)_{\alpha \gamma} d \widetilde{g}^{\gamma \beta}+\left(\widetilde{g}^{-1}\right)_{\rho \gamma} \pi_{\mathbb{P} M}^{*} \Omega_{\sigma i}^{\rho} \mathbf{G}_{\alpha}^{\gamma} \widetilde{g}^{\sigma \beta} \otimes d x^{i} .
$$

If $M$ is $n$-dimensional, the tangent space $T_{p}(\mathbb{P})$ is $(n+d)$-dimensional. Since the vertical subspace $V_{p}(\mathbb{P})$ is tangential to the fiber $G$, it is $d$-dimensional. Accordingly, $H_{p}(\mathbb{P})$ is $n$-dimensional. The basis of $V_{p}(\mathbb{P})$ can be taken to be $\partial_{\alpha \beta}:=\frac{\partial}{\partial g^{\alpha \beta}}$. Now, let the basis of $H_{p}(\mathbb{P})$ be denoted by

$$
E_{i}:=\partial_{i}+\Gamma_{i}^{\alpha \beta} \partial_{\alpha \beta}, i=1,2,3, . . n \text { and } \alpha, \beta=1,2,3, . . d
$$

where $\partial_{i}=\frac{\partial}{\partial x^{i}}$. The connection 1 -form $\omega$ projects $T_{p}(\mathbb{P})$ onto $V_{p}(\mathbb{P})$. In order for $X \in T_{p}(\mathbb{P})$ to belong to $H_{p}(\mathbb{P})$, it has to be $X \in H_{p}(\mathbb{P}), \omega_{p}(X)=\langle\omega(p) \mid X\rangle=0$. In other words,

$$
H_{p}(\mathbb{P}):=\left\{X \in T_{p}(\mathbb{P}) \mid \omega_{p}(X)=0\right\},
$$

from which $\Omega_{i}^{\alpha \beta}$ can be determined. The inner product appearing in $\omega_{p}(X)=\langle\omega(p) \mid X\rangle=0$ is a map $\langle\cdot \mid \cdot\rangle: T_{p}^{*}(\mathbb{P}) \times T_{p}(\mathbb{P}) \rightarrow \mathbb{R}$ defined by $\langle W \mid V\rangle=$ $W_{\mu} V^{\nu}\left\langle d x^{\mu} \mid \frac{\partial}{\partial x^{\nu}}\right\rangle=W_{\mu} V^{\nu} \delta_{\nu}^{\mu}$, where the 1-form $W$ and vector $V$ are given by $W=W_{\mu} d x^{\mu}$ and $V=V^{\mu} \frac{\partial}{\partial x^{\nu}}$. Observe also that, $\left\langle d g^{\alpha \beta} \mid \partial_{\rho \sigma}\right\rangle=\delta_{\rho}^{\alpha} \delta_{\sigma}^{\beta}$.

We parameterize an arbitrary group element $\widetilde{g}_{\lambda}$ as $\widetilde{g}(\lambda)=e^{\lambda^{A} \mathbf{G}_{A}}=e^{\lambda \cdot \mathbf{G}}, A=1, . . \operatorname{dim}(\mathfrak{g})$. The right action $R_{\widetilde{g}(\lambda)}=R_{\exp (\lambda \cdot G)}$ on $p \in \mathbb{P}$, i.e. $R_{\exp (\lambda \cdot \mathbf{G})} p=$ $p \exp (\lambda \cdot \mathbf{G})$, defines a curve through $p$ in $\mathbb{P}$. Define a vector $G^{\#} \in T_{p}(\mathbb{P})$ by 53 .

$$
G^{\#} f(p):=\left.\frac{d}{d t} f(p \exp (\lambda \cdot \mathbf{G}))\right|_{\lambda=0},
$$

where $f: \mathbb{P} \rightarrow \mathbb{R}$ is an arbitrary smooth function. Since the vector $G^{\#}$ is tangent to $\mathbb{P}$ at $p, G^{\#} \in V_{p}(\mathbb{P})$, the components of the vector $G^{\#}$ are the fundamental vector fields at $p$ which constitute $V(\mathbb{P})$. We have to stress that the components of $G^{\#}$ may also be viewed as a basis element of the Lie algebra $\mathfrak{g}$. Given $G^{\#} \in V_{p}(\mathbb{P}), \mathbf{G} \in \mathfrak{g}$,

$$
\begin{aligned}
\omega_{p}\left(G^{\#}\right) & =\left\langle\omega(p) \mid G^{\#}\right\rangle=\widetilde{g}^{-1} d \widetilde{g}\left(G^{\#}\right)+\widetilde{g}^{-1} \pi_{\mathbb{P} M}^{*} \Omega \widetilde{g}\left(G^{\#}\right) \\
& =\left.\widetilde{g}_{p}^{-1} \widetilde{g}_{p} \frac{d}{d \lambda}(\exp (\lambda \cdot \mathbf{G}))\right|_{\lambda=0},
\end{aligned}
$$

where use was made of $\pi_{\mathbb{P} M *} G^{\#}=0$. Hence, $\omega_{p}\left(G^{\#}\right)=$ G. An arbitrary vector $X \in H_{p}(\mathbb{P})$ may be expanded in a basis spanning $H_{p}(\mathbb{P})$ as $X:=\beta^{i} E_{i}$. By direct computation, one can show

$$
\begin{aligned}
\left\langle\omega_{\alpha}^{\beta} \mid X\right\rangle & =\left(\widetilde{g}^{-1}\right)_{\alpha \gamma} \beta^{i} \Gamma_{i}^{\gamma \beta}+ \\
+ & \left(\widetilde{g}^{-1}\right)_{\alpha \gamma} \pi_{\mathbb{P} M}^{*} \Omega_{\sigma i}^{\rho} \beta^{i} \mathbf{G}_{\rho}^{\gamma} \widetilde{g}^{\sigma \beta}=0, \forall \beta^{i}
\end{aligned}
$$

Equation (18) yields

$$
\left(\widetilde{g}^{-1}\right)_{\alpha \gamma} \Gamma_{i}^{\gamma \beta}+\left(\widetilde{g}^{-1}\right)_{\alpha \gamma} \pi_{\mathbb{P} M}^{*} \Omega^{\rho}{ }_{\sigma i} \mathbf{G}_{\rho}^{\gamma} \widetilde{g}^{\sigma \beta}=0,
$$


from which we obtain

$$
\Gamma_{i}^{\gamma \beta}=-\pi_{\mathbb{P} M}^{*} \Omega_{\sigma i}^{\rho} \mathbf{G}_{\rho}^{\gamma} \widetilde{g}^{\sigma \beta} .
$$

In this manner, the horizontal component is completely determined. An arbitrary tangent vector $\mathfrak{X} \in T_{p}(\mathbb{P})$ defined at $p \in \mathbb{P}$ takes the form

$$
\mathfrak{X}=A^{\alpha \beta} \partial_{\alpha \beta}+B^{i}\left(\partial_{i}-\pi_{\mathbb{P} M}^{*} \Omega^{\rho}{ }_{\sigma i} \mathbf{G}_{\rho}^{\alpha} \widetilde{g}^{\sigma \beta} \partial_{\alpha \beta}\right),
$$

where $A^{\alpha \beta}$ and $B^{i}$ are constants. The vector field $\mathfrak{X}$ is comprised of horizontal $\mathfrak{X}^{H}:=$ $B^{i}\left(\partial_{i}-\pi_{\mathbb{P} M}^{*} \Omega^{\rho}{ }_{\sigma i} \mathbf{G}_{\rho}^{\alpha} \widetilde{g}^{\sigma \beta} \partial_{\alpha \beta}\right) \in H(\mathbb{P})$ and vertical $\mathfrak{X}^{V}:=$ $A^{\alpha \beta} \partial_{\alpha \beta} \in V(\mathbb{P})$ components.

Let $\mathfrak{X} \in T_{p}(\mathbb{P})$ and $g \in \mathbf{G}$, then

$$
\begin{aligned}
R_{g}^{*} \omega(\mathfrak{X})= & \omega\left(R_{g *} \mathfrak{X}\right)= \\
& =\widetilde{g}_{p g}^{-1} \Omega\left(R_{g *} \mathfrak{X}\right) \widetilde{g}_{p g}+\widetilde{g}_{p g}^{-1} d \widetilde{g}_{p g}\left(R_{g *} \mathfrak{X}\right),
\end{aligned}
$$

Observing that $\widetilde{g}_{p g}=\widetilde{g}_{p} g$ and $\widetilde{g}_{g p}^{-1}=g^{-1} \widetilde{g}_{p}^{-1}$ the first term on the RHS of (22) reduces to $\widetilde{g}_{p g}^{-1} \Omega\left(R_{g_{*}} \mathfrak{X}\right) \widetilde{g}_{p g}=g^{-1} \widetilde{g}_{p}^{-1} \Omega\left(R_{g *} \mathfrak{X}\right) \widetilde{g}_{p} g$ while the second term gives $\widetilde{g}_{p g}^{-1} d \widetilde{g}_{p g}\left(R_{g *} \mathfrak{X}\right)=g^{-1} \widetilde{g}_{p}^{-1} d\left(R_{g *} \mathfrak{X}\right) \widetilde{g}_{p} g$. We therefore conclude

$$
R_{g}^{*} \omega_{\lambda}=a d_{g^{-1}} \omega_{\lambda},
$$

where the adjoint map ad is defined by

$$
\begin{aligned}
& a d_{g} Y:=L_{g^{*}} \circ R_{g^{-1} *} \circ Y=g Y g^{-1}, \\
& a d_{g^{-1}} Y:=g^{-1} Y g .
\end{aligned}
$$

The potential $\Omega^{A}$ can be obtained from $\omega$ as $\Omega^{A}=$ $s^{*} \omega$. To demonstrate this, let $Y \in T_{p}(M)$ and $\widetilde{g}$ be specified by the inverse diffeomorphism or trivialization map (9) with $\chi_{\lambda}^{-1}(p)=\left(x, \widetilde{g}_{\lambda}\right)$ for $p(x)=s_{\lambda}(x) \cdot \widetilde{g}_{\lambda}$. We find $s_{i}^{*} \omega(Y)=\widetilde{g}^{-1} \Omega\left(\pi_{*} s_{i *} Y\right) \widetilde{g}+\widetilde{g}^{-1} d \widetilde{g}\left(s_{i *} Y\right)$, where we have used $s_{i *} Y \in T_{s_{i}}(\mathbb{P}), \pi_{*} s_{i *}=(i d)_{T_{p}(M)}$ and $\widetilde{g}=$ $(i d)_{G}$ at $s_{i}$ implying $\widetilde{g}^{-1} d \widetilde{g}\left(s_{i *} Y\right)=0$ [53]. Hence,

$$
s_{i}^{*} \omega(Y)=\Omega(Y) .
$$

To determine the gauge transformation of the connection 1-form $\omega$, we use the fact that $R_{\widetilde{g} *} X=X \widetilde{g}$ for $X \in T_{p}(M)$ and the transition functions $\widetilde{g}_{n m} \in G$ defined between neighboring bundle charts (66). By direct computation we get

$$
\begin{aligned}
c_{j *} X & =\left.\frac{d}{d t} c_{j}(\lambda(t))\right|_{t=0}=\left.\frac{d}{d t}\left[c_{i}(\lambda(t)) \cdot \widetilde{g}_{i j}\right]\right|_{t=0} \\
& =R_{\widetilde{g}_{i j} *} c_{i}^{*}(X)+\left(\widetilde{g}_{j i}^{-1}(x) d \widetilde{g}_{i j}(X)\right)^{\#},
\end{aligned}
$$

where $\lambda(t)$ is a curve in $M$ with boundary values $\lambda(0)=$ $m$ and $\left.\frac{d}{d t} \lambda(t)\right|_{t=0}=X$. Thus, we obtain the useful result

$$
c_{*} X=R_{\widetilde{g} *}\left(c_{*} X\right)+\left(\widetilde{g}^{-1} d \widetilde{g}(X)\right)^{\#} .
$$

Applying $\omega$ to Eq. (27), we get

$$
\omega\left(c_{*} X\right)=c^{*} \omega(X)=a d_{\widetilde{g}^{-1}} c^{*} \omega(X)+\widetilde{g}^{-1} d \widetilde{g}(X), \forall X .
$$

Hence, the gauge transformation of the local gauge potential $\Omega$ reads,

$$
\Omega \rightarrow \Omega^{\prime}=a d_{\widetilde{g}^{-1}}(d+\Omega)=\widetilde{g}^{-1}(d+\Omega) \widetilde{g} .
$$

Since $\Omega=c^{*} \omega$ we obtain, from Eq.(29), the gauge transformation law of $\omega$

$$
\omega \rightarrow \omega^{\prime}=\widetilde{g}^{-1}(d+\omega) \widetilde{g} .
$$

We have now all the ingredients to investigate the bundle structure of the gravitation field.

\section{B. The Bundle Structure for Gravitation}

Let us recall the definition of gauge transformations in the context of ordinary fiber bundles. Given a principal fiber bundle $\mathbb{P}(M, G ; \pi)$ with base space $M$ and standard $G$-diffeomorphic fiber, gauge transformations are characterized by bundle isomorphisms $\lambda: \mathbb{P} \rightarrow \mathbb{P}$ exhausting all diffeomorphisms $\lambda_{M}$ on $M$ [54]. This mapping is called an automorphism of $\mathbb{P}$ provided it is equivariant with respect to the action of $G$. This amounts to restrict the action $\lambda$ of $G$ along local fibers leaving the base space unaffected. Indeed, with regard to gauge theories of internal symmetry groups, a gauge transformation is a fiber preserving bundle automorphism, i.e. diffeomorphisms $\lambda$ with $\lambda_{M}=(i d)_{M}$. The automorphisms $\lambda$ form a group called the automorphism group $A u t_{\mathbb{P}}$ of $\mathbb{P}$. The gauge transformations form a subgroup of $A u t_{\mathbb{P}}$ called the gauge group $G\left(A u t_{\mathbb{P}}\right)$ (or $G$ in short) of $\mathbb{P}$.

The map $\lambda$ is required to satisfy two conditions, namely its commutability with the right action of $G$ [the equivariance condition $\left.\lambda\left(R_{g}(p)\right)=\lambda(p g)=\lambda(p) g\right]$

$$
\lambda \circ R_{g}(p)=R_{g}(p) \circ \lambda, \quad p \in \mathbb{P}, g \in G
$$

according to which fibers are mapped into fibers, and the verticality condition

$$
\pi \circ \lambda(u)=\pi(u)
$$

where $u$ and $\lambda(u)$ belong to the same fiber. The last condition ensures that no diffeomorphisms $\lambda_{M}: M \rightarrow M$ given by

$$
\lambda_{M} \circ \pi(u)=\pi \circ \lambda(u),
$$

be allowed on the base space $M$. In a gauge description of gravitation, one is interested in gauging external transformation groups. This means that the group action on space-time coordinates cannot be neglected. The spaces of internal fiber and external base must be interlocked in the sense that transformations in one space must induce corresponding transformations in the other. The usual definition of a gauge transformation, i.e. as a displacement along local fibers not affecting the base space, must be generalized to reflect this interlocking. One possible way of framing this interlocking is to employ a nonlinear realization of the gauge group $G$, provided a closed 
subgroup $H \subset G$ exists. The interlocking requirement is then transformed into the interplay between groups $G$ and one of its closed subgroups $H$.

Let us denote by $G$ a Lie group with elements $\{g\}$. Let $H$ be a closed subgroup of $G$ specified by

$$
H:=\left\{h \in G \mid \Pi\left(R_{h} g\right)=\pi(g), \forall g \in G\right\},
$$

with elements $\{h\}$ and known linear representations $\rho(h)$. Here $\Pi$ is a projection map and $R_{h}$ is the right group action. Let $M$ be a differentiable manifold with points $\{x\}$ to which $G$ and $H$ may be referred, i.e. $g=g(x)$ and $h=h(x)$. Being that $G$ and $H$ are Lie groups, they are also manifolds. The right action of $H$ on $G$ induces a complete partition of $G$ into mutually disjoint orbits $g H$. Since $g=g(x)$, all elements of $g H=\left\{g h_{1}, g h_{2}, g h_{3}, \cdots, g h_{n}\right\}$ are defined over the same $x$. Thus, each orbit $g H$ constitutes an equivalence class of point $x$, with equivalence relation $g \equiv g^{\prime}$ where $g^{\prime}=R_{h} g=g h$.

By projecting each equivalence class onto a single element of the quotient space $\mathcal{M}:=G / H$, the group $G$ becomes organized as a fiber bundle in the sense that $G=\bigcup_{i}\left\{g_{i} H\right\}$. In this manner the manifold $G$ is viewed as a fiber bundle $G(\mathcal{M}, H$; $\Pi)$ with $H$-diffeomorphic fibers $\Pi^{-1}(\xi): G \rightarrow \mathcal{M}=g H$ and base space $\mathcal{M}$. A composite principal fiber bundle $\mathbb{P}(M, G ; \pi)$ is one whose $G$-diffeomorphic fibers possess the fibered structure $G(\mathcal{M}, H ; \Pi) \simeq \mathcal{M} \times H$ described above. The bundle $\mathbb{P}$ is then locally isomorphic to $M \times G(\mathcal{M}, H)$. Moreover, since an element $g \in G$ is locally homeomorphic to $\mathcal{M} \times H$ the elements of $\mathbb{P}$ are - by transitivity - also locally homeomorphic to $M \times \mathcal{M} \times H \simeq \Sigma \times H$ where (locally) $\Sigma \simeq M \times \mathcal{M}$. Thus, an alternative view of $\mathbb{P}(M, G$; $\pi)$ is provided by the $\mathbb{P}$-associated $H$-bundle $\mathbb{P}(\Sigma, H ; \widetilde{\pi})$ [37]. The total space $\mathbb{P}$ may be regarded as $G(\mathcal{M}, H ; \Pi)$ bundles over the base space $M$ or equivalently as $H$-fibers attached to the manifold $\Sigma \simeq M \times \mathcal{M}$.

The nonlinear realization (NLR) technique 21, 22] provides a way to determine the transformation properties of fields defined on the quotient space $G / H$. The NLR of $\operatorname{Diff}(4, \mathbb{R})$ becomes tractable due to a theorem by Ogievetsky. According to this theorem [24], the algebra of the infinite dimensional group $\operatorname{Diff}(4, \mathbb{R})$ can be taken as the closure of the finite dimensional algebras of $S O(4$, $2)$ and $A(4, \mathbb{R})$. Remind that the Lorentz group generates transformations that preserve the quadratic form on Minkowski space-time built from the metric tensor, while the special conformal group generates infinitesimal anglepreserving transformations on Minkowski space-time.

The affine group is a generalization of the Poincaré group where the Lorentz group is replaced by the group of general linear transformations. As such, the affine group generates translations, Lorentz transformations, volume preserving shear and volume changing dilation transformations. As a consequence, the $\operatorname{NLR}$ of $\operatorname{Diff}(4, \mathbb{R}) / S O(3$, 1) can be constructed by taking a simultaneous realization of the conformal group $S O(4,2)$ and the affine group $A(4, \mathbb{R}):=\mathbb{R}^{4} \rtimes G L(4, \mathbb{R})$ on the coset spaces $A(4$,
$\mathbb{R}) / S O(3,1)$ and $S O(4,2) / S O(3,1)$. One possible interpretation of this theorem is that the conformal-affine group CA (defined below) may be the largest subgroup of $\operatorname{Diff}(4, \mathbb{R})$, whose transformations may be put into the form of a generalized coordinate transformation. We remark that a NLR can be made linear by embedding the representation in a sufficiently higher dimensional space. Alternatively, a linear group realization becomes nonlinear when subject to constraints. One type of relevant constraints may be those responsible for symmetry reduction from $\operatorname{Diff}(4, \mathbb{R})$ to $S O(3,1)$ for instance.

We take the group $C A(3,1)$ as the basic symmetry group $G$. The CA group consists of the groups $S O(4$, 2 ) and $A(4, \mathbb{R})$. In particular, $\mathrm{CA}$ is proportional to the union $S O(4,2) \cup A(4, \mathbb{R})$. We know however that the affine and special conformal groups have several group generators in common. These common generators reside in the intersection $S O(4,2) \cap A(4, \mathbb{R})$ of the two groups, within which there are two copies of $\Pi:=D \times P(3,1)$, where $D$ is the group of scale transformations (dilations) and $P(3,1):=T(3,1) \rtimes S O(3,1)$ is the Poincaré group.

Finally we define the CA group as the union of the affine and conformal groups minus one copy of the overlap $\Pi$, i.e. $C A(3,1):=S O(4,2) \cup A(4, \mathbb{R})-\Pi$. Being defined in this way we recognize that $C A(3,1)$ is a 24 parameter Lie group representing the action of Lorentz transformations (6), translations (4), special conformal transformations (4), space-time shears (9) and scale transformations (1). All these transformations can be adopted to define any conformally-affine theory of gravity.

In this paper, we obtain the NLR of $C A(3,1)$ modulo $S O(3,1)$ as $4 \mathrm{D}$ realizations starting from $5 \mathrm{D}$-manifold [3]. This procedure has been recently adopted also in holographic approaches to Quantum Chromodynamics [91].

\section{THE GROUP STRUCTURE IN 5D AND 4D-SPACES}

In this section, we will discuss the group structure of a 5D-Riemannian manifold (in particular the Lorentz group) and its reduction to 4D-manifold. Such an approach gives a useful tool to deal with the realization of effective theories of gravity in $4 \mathrm{D}$ and the problem of mass generation. Let us start with the necessary definition of the Minkowski space-time $\mathbf{R}^{4}$ endowed with the metric

$$
(X, X)_{4}=\left(x^{0}\right)^{2}-\vec{X} \cdot \vec{X},
$$

where $X=\left(x^{0}, \vec{X}\right)$ is a four-vector, $x^{0}$ is the time coordinate and $\vec{X}$ is an ordinary vector in $\mathbf{R}^{3}$. The Lorentz transformations are those linear transformations of Minkowski-type space that leave $(X, X)_{4}$, the scalar product of four-vectors, invariant:

$$
X \longrightarrow X^{\prime}=\Lambda X,
$$


being $(\Lambda X, \Lambda X)=(X, X)$. If $g$ is the Minkowski metric with signature $(+---), \Lambda$ is a Lorentz transformation when $\Lambda^{t} g \Lambda=g$. The set of such transformations is the orthogonal group $O(4)$, namely $O(1,3)$ considering the time-like and space-like components, group characterized, as well known, by the properties that $\operatorname{det} \Lambda= \pm 1$ and the number of generators is 6 . The coset decomposition of such a group is

$O(1,3)=\mathbf{I} S O(1,3) \cup \Lambda_{P} S O(1,3) \cup \Lambda_{T} S O(1,3) \cup \Lambda_{P T}(1,3)$,

where $\mathbf{I} S O(1,3)$ is the proper orthochronus Lorentz group with $\operatorname{det} \Lambda=1$, whose elements preserve parity (spatial orientation) and the direction of time; $\Lambda_{P} S O(1,3)$ is the group of spatial inversion (parity inversion); $\Lambda_{T} S O(1,3)$ are the time reversal transformations and $\Lambda_{P T}(1,3)$ are the total space-time inversion, where we have taken into account all the components without arbitrarily discarding any part of them. The covering group of $S O(1,3)$ is the simply connected complex group $S L(2, C)$ whose physical meaning is that particles (or in general fields) transform according to its representations.

Now we want to extend this scheme to a $5 \mathrm{D}$-space (which we, initially, consider a flat manifold), where we do not define a priori a signature for the metric and which, after a $4 \mathrm{D}$-reduction procedure, must be capable of reproducing all the features of Lorentz group. For the sake of generality, we do not specify the signature and the number of dimensions. Below we will assume $N=5$.

Let $\mathcal{M}^{(p, q)}=\mathbf{R}^{N}$ be a manifold where $p, q \geq 0$ are integers and such that $p+q=N$ with the flat metric $d S^{2}={ }^{(p, q)} \eta_{A B} d x^{A} d x^{B}$ and $A, B=0,1,2, \ldots, N-1$. A general signature is

$$
{ }^{(p, q)} \eta=\left({ }^{(p, q)} \eta_{A B}\right)=\operatorname{diag}(\underbrace{1,1, . ., 1}_{p} ; \underbrace{-1,-1, . .,-1}_{q}),
$$

where $p$ are the time-like directions and $q$ are the spacelike directions. As particular cases, we have

$$
\begin{aligned}
\mathbf{E}^{N}=\mathcal{M}^{(0, N)} & =\text { Euclidean space, } \\
\mathcal{M}^{N}=\mathcal{M}^{(1, N-1)} & =N \text {-dimensional Minkowski space. }
\end{aligned}
$$

It is important to stress that the other flat (pseudo)Riemannian spaces have more than one equivalent (independent) time-like directions and hence have no distinction between future and past time-like directions as they have in Minkowski space. This fact means that the spacelike pseudo-spheres are connected hypersurfaces, rather than having two disjoint components as in Minkowski space. The metric can be written as

$$
d S^{2}=\left(\sum_{A, B=0}^{p} \delta_{A B} d x^{A} d x^{B}\right)-\left(\sum_{A, B=p+1}^{N-1} \delta_{A B} d x^{A} d x^{B}\right)
$$

where time-like and space-like components are clearly separated. Some considerations are necessary at this point. The metric (39) is invariant under rotations of the time-like directions among themselves (except for $E^{N}$ and $\mathcal{M}^{N}$ which are degenerate particular cases, since in the first case there are no time arrows and in the second case, only one time arrow exists by definition) and of the space-like directions among themselves. The remaining independent pseudo-rotations are all boosts each involving a time-like and a space-like direction. The physical meaning of such a result is that close time-like paths are an usual feature in pseudo-Riemannian manifolds, moreover a definite time arrow distinguishing the past from the future is only a particular characteristic of Minkowski spaces where Lorentz transformations work.

Let us now take into account the possible linear transformations on this $\mathcal{M}^{(p, q)}$-manifold. A pseudoorthogonal group $O(p, q)$ can be defined on this pseudoRiemannian manifold. This group consists of all the linear transformations $X^{A} \rightarrow \Lambda_{B}^{A} X^{B}$ such that the metric (39) is invariant, i.e.

$$
d S^{2} \longrightarrow \eta_{A B} \Lambda_{C}^{A} \Lambda_{D}^{B} d x^{C} d x^{D}=\eta_{C D} d x^{C} d x^{D},
$$

more precisely we can say that

$$
O(p, q) \equiv\left\{\left(\Lambda_{B}^{A}\right) \in G L(N, \mathbf{R}) \mid \eta_{A B} \Lambda_{C}^{A} \Lambda_{D}^{B}=\eta_{C D}\right\},
$$

where $G L(N, \mathbf{R})$ are non-singular matrices in $N$ dimensions. Note that

$$
\operatorname{det}\left(\eta_{A B}\right)\left[\operatorname{det}\left(\Lambda_{B}^{A}\right)\right]^{2}=\operatorname{det}\left(\eta_{A B}\right) \longrightarrow \operatorname{det}\left(\Lambda_{B}^{A}\right)= \pm 1,
$$

where the determinant is +1 for rotations $S O(p, q)$ and -1 for inversions, inversions which do not constitute a sub-group (the product of two inversions gives a rotation). In the first case, we have

$$
S O(p, q) \equiv\left\{\left(\Lambda_{B}^{A}\right) \in O(p, q) \mid \operatorname{det}\left(\Lambda_{B}^{A}\right)=1\right\},
$$

which is a special pseudo-orthogonal group. An important feature of such a group is that it consists of two disconnected pieces when both $p$ and $q$ are odd (see [55] for the general demonstration). Special examples of $S O(p, q)$ are

$$
\begin{aligned}
& S O(0, N) \equiv S O(N, \mathbf{R}) \\
& \text { for } p=0 \quad \text { special orthogonal group, } \\
& S O(1, N-1) \\
& \text { for } \quad p=1 \quad \text { Lorentz or De Sitter group. }
\end{aligned}
$$

The group $S O(p, q)$ can be decomposed as follows

$$
S O(p, q)=\left(\begin{array}{ccc}
\overbrace{S O(p, \mathbf{R})}^{p \times p} & \overbrace{\text { boosts }}^{p \times q} \\
---- & ---- \\
\underbrace{\text { boosts }}_{q \times p} & \mid \underbrace{S O(q, \mathbf{R})}_{q \times q}
\end{array}\right),
$$

where $S O(p, \mathbf{R})$ are $(p \times p)$ square matrices which rotate the time-like directions among themselves, $S O(q, \mathbf{R})$ are 
$(q \times q)$ square matrices which rotate the space-like directions among themselves, and the boosts are, in general, $(p \times q)$ or $(q \times p)$ rectangular matrices which rotate timelike and space-like directions.

The number of generators of the $S O(p, q)$ group, i.e. the number of independent elements or the dimension of the group, can be easily calculated being, in general,

$$
\operatorname{dim} S O(N)=\frac{N(N-1)}{2} .
$$

An important remark is in order at this point. It is wellknown, since an old result by Riemann [56], that a Ndimensional metric has $s=N(N-1) / 2$ degrees of freedom, that is, it is locally equivalent to give $s$ independent functions. This feature is related to the choice of local charts but it is also related to the number of degrees of freedom of gravitational field. As we will discuss below, this is a key ingredient of our discussion. In our case, we have

$$
\operatorname{dim} S O(p, q)=\operatorname{dim} S O(p, \mathbf{R})+\operatorname{dim} S O(q, \mathbf{R})+p \cdot q .
$$

The result is

$$
\frac{N(N-1)}{2}=\frac{p(p-1)}{2}+\frac{q(q-1)}{2}+p \cdot q,
$$

where $p \cdot q$ is the number of independent pairs of one space-like and one time-like direction. For $N=5$, we have 10 independent elements.

Clearly the rotations $S O(p, \mathbf{R}) \otimes S O(q, \mathbf{R})$ form a subgroup of $S O(p, q)$ but the boosts do not; boosts along different directions combine to give a boost plus a rotation.

Let us now add the $N$ translations $\tilde{X}^{A} \rightarrow X^{A}+a^{A}$ to the pseudo-orthogonal group $O(p, q)$, consisting of rotations and inversions. This fact yields the full group of motions of $\mathcal{M}^{(p, q)}$, which can be classified in the most general inhomogeneous pseudo-orthogonal group $I O(p, q)$. Not taking into account the inversions, a remarkable subgroup is $I S O(p, q)$, the inhomogeneous special pseudoorthogonal group, of dimension

$$
r=\frac{N(N-1)}{2}+N=\frac{N(N+1)}{2},
$$

which gives $r=15$ for $N=5$. More generally, these groups can be realized as matrix groups in $N+1$ dimensions by adding a trivial row and a nontrivial column to $O(p, q)$, i.e.

$$
I O(p, q)=\left(\begin{array}{c|c}
O(p, q) & a^{0} \\
& \cdot \\
---- & a^{N-1} \\
0 \cdots 0 & 1
\end{array}\right)
$$

This fact is extremely interesting for our purposes since, adding up a dimension to the manifold in which we define dynamics allows to remove the singularities. As special cases, we have

- $I O(0, N)=I O(N, \mathbf{R})=$

Euclidean group in $N$ dimensions,

- $I O(1, N-1)=$

Poincaré group in $N$ dimensions,

or inhomogeneous Lorentz group.

The pseudo-spheres at the origin of $\mathcal{M}^{(p, q)}$ satisfy the fundamental relation

$$
\eta_{A B} x^{A} x^{B}=\text { constant } .
$$

Connected components of pseudo-sphere are $(N-1)$ dimensional hypersurfaces on which acts a $N(N-1) / 2-$ dimensional group, under which all points are equivalent. Such spaces are all of constant curvature and of different signatures (which are determined by considering coordinate directions at their intersection with the Cartesian axes; property which is important for the following projection process).

This general discussion can be specialized to the 5Dcase. First of all, we assume that a 5D-vector field defines a metric whose signature is given by

$$
(X, X)_{5}=\left(x^{0}\right)^{2}-\vec{X} \cdot \vec{X}+\varepsilon\left(x_{4}\right)^{2},
$$

where $\varepsilon= \pm 1$, so that, using the traditional terms, the fifth dimension can be time-like or space-like. Moreover, as we shall see below, it is the $4 \mathrm{D}$-dynamics which discriminates, by a bijective correspondence, the signature giving rise to particle-like solutions $(\varepsilon=-1)$ or to wavelike solutions $(\varepsilon=+1)$. When $N=5$, we can obtain pseudo-spheres of Lorentz signature and thus 4D-spacetimes of constant curvature (as Friedmann-RobertsonWalker ones). There are only two independent different signatures for $N=5$. They are $(p, q)=(1,4)$, corresponding to the case $\varepsilon=-1$ and $(p, q)=(2,3)$, corresponding to $\varepsilon=+1$. The $5 \mathrm{D}$-manifolds are $\mathcal{M}^{(1,4)}=\mathbf{R}^{5}$ and $\mathcal{M}^{(2,3)}=\mathbf{R}^{5}$, respectively, where $\mathbf{R}^{5}$ is the $5 \mathrm{D}$-space. As it is well-known, the former case is called the De Sitter space, while the latter is the Anti-De Sitter one. The fact that the standard signature of the universe is $(+---)$ can be derived from an equivalent process starting from $\mathcal{M}^{(1,4)}$ or $\mathcal{M}^{(2,3)}$. The discrimination is dynamically achieved, as we shall see below, when particle masses, after the embedding, spring out. Due to this fact, we are going to deal with the degrees of freedom of the space-time and of the particles under the same standard so that a straightforward decomposition of our 5D-group can be

$$
\mathcal{G}_{5} \supset I O(3,1) \otimes S U(3) \otimes S U(2) \otimes U(1),
$$


and then if $\mathcal{G}_{5}=S L(5)$, this is the minimal group, with $N^{2}-1=24$ parameters which is capable of including all the standard fundamental interactions and the 10 generators of inhomogeneous Lorentz group. In particular, it can include the inhomogeneous pseudo-orthogonal group $I O(5)$ (in the two modalities $I O(1,4)$ or $I O(2,3)$ ), which is a sub-group of $S L(5)$, comprehensive of all space-time rotations, inversions and translations in $5 \mathrm{D}$. It is clear that 4D pseudo-Riemannian manifolds $\mathcal{M}^{(1,3)}$ can be obtained from both $\mathcal{M}^{(1,4)}$ or $\mathcal{M}^{(2,3)}$. This reduction procedure, as we will show below, is a dynamical process depending on the splitting of the 5D-field equations.

An important consideration is necessary at this point. After the reduction to 4D-physics, a given theory of gravity is described by the conformal-affine group of diffeomorphisms $G L(4)$ which is characterized by $4 \times 4=16$ generators. A straightforward splitting of such a group is

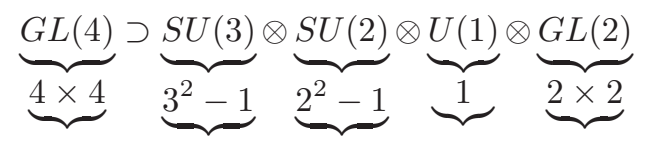

where the number of generators is indicated for any subgroup. The physical meaning of $S U(3), S U(2)$ and $U(1)$ is clear while $G L(2)$ represents a group of diffeomorphisms with 4 generators. The further generators of gravitational field can be recovered in the framework of Extended Theories of Gravity [49, 50] as we will see below. The experimental consequences of these further gravitational modes could be extremely interesting for the physics at LHC.

\section{SPACE-TIME DEFORMATIONS AND THE GL(4)-GROUP}

Another ingredient for our considerations is represented by the space-time deformations that are elements of the $G L(4)$-group. Let us take into account a metric $\mathbf{g}$ on a space-time manifold $\mathcal{M}$. Such a metric is assumed to be an exact solution of the gravitational field equations. From the discussion in Sec. III, we can decompose it by a co-tetrad field $\omega^{A}(x)$

$$
\mathbf{g}=\eta_{A B} \omega^{A} \omega^{B} .
$$

Let us define now a new tetrad field $\widetilde{\omega}=\Phi^{A}{ }_{C}(x) \omega^{C}$, with $\Phi^{A}{ }_{C}(x)$ a matrix of scalar fields. Finally we introduce a space-time $\widetilde{\mathcal{M}}$ with the metric $\widetilde{g}$ defined in the following way

$$
\widetilde{\mathbf{g}}=\eta_{A B} \Phi_{C}^{A} \Phi^{B}{ }_{D} \omega^{C} \omega^{D}=\gamma_{C D}(x) \omega^{C} \omega^{D}
$$

where also $\gamma_{C D}(x)$ is a matrix of fields which are scalars with respect to the coordinate transformations.

If $\Phi_{C}^{A}(x)$ is a Lorentz matrix in any point of $\mathcal{M}$, then

$$
\widetilde{g} \equiv g
$$

otherwise we say that $\widetilde{g}$ is a deformation of $g$ and $\widetilde{\mathcal{M}}$ is a deformed $\mathcal{M}$. If all the functions of $\Phi^{A}{ }_{C}(x)$ are continuous, then there is a one - to - one correspondence between the points of $\mathcal{M}$ and the points of $\widetilde{\mathcal{M}}$. If $\xi$ is a Killing vector for $g$ on $\mathcal{M}$, the corresponding vector $\widetilde{\xi}$ on $\widetilde{\mathcal{M}}$ could not necessarily be a Killing vector.

A particular subset of these deformation matrices is given by

$$
\Phi_{C}^{A}(x)=\Omega(x) \delta^{A}{ }_{C}
$$

which define conformal transformations of the metric,

$$
\widetilde{g}=\Omega^{2}(x) g .
$$

In this sense, the deformations defined by Eq. (54) can be regarded as a generalization of the conformal transformations which we will discuss below.

We call the matrices $\Phi_{C}^{A}(x)$ first deformation matrices, while we can refer to

$$
\gamma_{C D}(x)=\eta_{A B} \Phi_{C}^{A}(x) \Phi^{B}{ }_{D}(x) .
$$

as the second deformation matrices, which, as seen above, are also matrices of scalar fields. They generalize the Minkowski matrix $\eta_{A B}$ with constant elements in the definition of the metric. A further restriction on the matrices $\Phi_{C}^{A}$ comes from the above mentioned theorem proved by Riemann by which an $N$-dimensional metric has $N(N-1) / 2$ degrees of freedom (see [58] for details).

With this definitions in mind, let us consider the main properties of deforming matrices. Let us take into account a four dimensional space-time with Lorentzian signature. A family of matrices $\Phi^{A}{ }_{C}(x)$ such that

$$
\Phi_{C}^{A}(x) \in G L(4) \forall x,
$$

are defined on such a space-time. These functions are not necessarily continuous and can connect space-times with different topologies. A singular scalar field introduces a deformed manifold $\widetilde{\mathcal{M}}$ with a space-time singularity.

As it is well known, the Lorentz matrices $\Lambda_{C}^{A}$ leave the Minkowski metric invariant and then

$\mathrm{g}=\eta_{E F} \Lambda^{E}{ }_{A} \Lambda^{F}{ }_{B} \Phi^{A}{ }_{C} \Phi^{B}{ }_{D} \omega^{C} \omega^{D}=\eta_{A B} \Phi^{A}{ }_{C} \Phi^{B}{ }_{D} \omega^{C} \omega^{D}$.

It follows that $\Phi^{A}{ }_{C}$ give rise to right cosets of the Lorentz group, i.e. they are the elements of the quotient group $G L(4, \mathbf{R}) / S O(3,1)$. On the other hand, a right-multiplication of $\Phi^{A}$ by a Lorentz matrix induces a different deformation matrix.

The inverse deformed metric is

$$
\widetilde{g}^{\alpha \beta}=\eta^{C D} \Phi_{C}^{-1}{ }_{C}^{A} \Phi^{-1}{ }_{D} e_{A}^{\alpha} e_{B}^{\beta}
$$

where $\Phi^{-1}{ }_{C}^{A} \Phi^{C}{ }_{B}=\Phi^{A}{ }_{C} \Phi^{-1}{ }_{B}=\delta_{B}^{A}$.

Let us decompose now the matrix $\Phi_{A B}=\eta_{A C} \Phi^{C}{ }_{B}$ in its symmetric and antisymmetric parts

$$
\Phi_{A B}=\Phi_{(A B)}+\Phi_{[A B]}=\Omega \eta_{A B}+\Theta_{A B}+\phi_{A B}
$$


where $\Omega=\Phi_{A}^{A}, \Theta_{A B}$ is the traceless symmetric part and $\phi_{A B}$ is the skew symmetric part of the first deformation matrix respectively. Then standard conformal transformations are nothing else but deformations with $\Theta_{A B}=\phi_{A B}=0$ [57].

Finding the inverse matrix $\Phi^{-1^{A}}$ in terms of $\Omega, \Theta_{A B}$ and $\phi_{A B}$ is not immediate, but as above, it can be split in the three terms

$$
\Phi_{C}^{-1^{A}}=\alpha \delta_{C}^{A}+\Psi_{C}^{A}+\Sigma_{C}^{A}
$$

where $\alpha, \Psi_{C}^{A}$ and $\Sigma^{A}{ }_{C}$ are respectively the trace, the traceless symmetric part and the antisymmetric part of the inverse deformation matrix. The second deformation matrix, from the above decomposition, takes the form

$$
\gamma_{A B}=\eta_{C D}\left(\Omega \delta_{A}^{C}+\Theta_{A}^{C}{ }_{A}+\phi_{A}^{C}\right)\left(\Omega \delta_{B}^{D}+\Theta^{D}{ }_{B}+\phi_{B}^{D}\right)
$$

and then

$$
\begin{aligned}
\gamma_{A B}= & \Omega^{2} \eta_{A B}+2 \Omega \Theta_{A B}+\eta_{C D} \Theta^{C}{ }_{A} \Theta^{D}{ }_{B}+ \\
& +\eta_{C D}\left(\Theta^{C}{ }_{A} \phi^{D}{ }_{B}+\phi^{C}{ }_{A} \Theta^{D}{ }_{B}\right)+\eta_{C D} \phi^{C}{ }_{A} \phi^{D}{ }_{B} .
\end{aligned}
$$

In general, the deformed metric can be split as

$$
\tilde{g}_{\alpha \beta}=\Omega^{2} g_{\alpha \beta}+\gamma_{\alpha \beta}
$$

where

$$
\begin{aligned}
\gamma_{\alpha \beta}= & \left(2 \Omega \Theta_{A B}+\eta_{C D} \Theta^{C}{ }_{A} \Theta^{D}{ }_{B}+\eta_{C D}\left(\Theta^{C}{ }_{A} \phi^{D}{ }_{B}+\right.\right. \\
& \left.\left.+\phi^{C}{ }_{A} \Theta^{D}{ }_{B}\right)+\eta_{C D} \phi^{C}{ }_{A} \phi^{D}{ }_{B}\right) \omega_{\alpha}^{A} \omega_{\beta}^{B}
\end{aligned}
$$

In particular, if $\Theta_{A B}=0$, the deformed metric simplifies to

$$
\widetilde{g}_{\alpha \beta}=\Omega^{2} g_{\alpha \beta}+\eta_{C D} \phi_{A}^{C} \phi_{B}^{D} \omega_{\alpha}^{A} \omega_{\beta}^{B}
$$

and, if $\Omega=1$, the deformation of a metric consists in adding to the background metric a tensor $\gamma_{\alpha \beta}$. We have to remember that all these quantities are not independent as, by the theorem mentioned in [58], they have to form at most six independent functions in a four dimensional space-time. Similarly the controvariant deformed metric can be always decomposed in the following way

$$
\widetilde{g}^{\alpha \beta}=\alpha^{2} g^{\alpha \beta}+\lambda^{\alpha \beta}
$$

Let us find the relation between $\gamma_{\alpha \beta}$ and $\lambda^{\alpha \beta}$. By using $\widetilde{g_{\alpha \beta} \beta} \widetilde{g^{\beta \gamma}}=\delta_{\alpha}^{\gamma}$, we obtain

$$
\alpha^{2} \Omega^{2} \delta_{\alpha}^{\gamma}+\alpha^{2} \gamma_{\alpha}^{\gamma}+\Omega^{2} \lambda_{\alpha}^{\gamma}+\gamma_{\alpha \beta} \lambda^{\beta \gamma}=\delta_{\alpha}^{\gamma}
$$

if the deformations are conformal transformations, we have $\alpha=\Omega^{-1}$, so assuming such a condition, one obtain the following matrix equation

$$
\alpha^{2} \gamma_{\alpha}^{\gamma}+\Omega^{2} \lambda_{\alpha}^{\gamma}+\gamma_{\alpha \beta} \lambda^{\beta \gamma}=0
$$

and

$$
\left(\delta_{\alpha}^{\beta}+\Omega^{-2} \gamma_{\alpha}^{\beta}\right) \lambda_{\beta}^{\gamma}=-\Omega^{-4} \gamma_{\alpha}^{\gamma}
$$

and finally

$$
\lambda_{\beta}^{\gamma}=-\Omega^{-4}\left(\delta+\Omega^{-2} \gamma\right)_{\beta}^{-1 \alpha} \gamma_{\alpha}^{\gamma}
$$

where $\left(\delta+\Omega^{-2} \gamma\right)^{-1}$ is the inverse tensor of $\left(\delta_{\alpha}^{\beta}+\Omega^{-2} \gamma_{\alpha}^{\beta}\right)$.

To each matrix $\Phi^{A}{ }_{B}$, we can associate a $(1,1)$-tensor $\phi_{\beta}^{\alpha}$ defined by

$$
\phi_{\beta}^{\alpha}=\Phi_{B}^{A} \omega_{\beta}^{B} e_{A}^{\alpha}
$$

such that

$$
\widetilde{g}_{\alpha \beta}=g_{\gamma \delta} \phi_{\alpha}^{\gamma} \phi_{\beta}^{\delta} .
$$

Vice-versa from a $(1,1)$-tensor $\phi_{\beta}^{\alpha}$, we can define a matrix of scalar fields as

$$
\phi_{B}^{A}=\phi^{\alpha}{ }_{\beta} \omega_{\alpha}^{A} e_{B}^{\beta} .
$$

In summary, space-time deformations are endowed with the conformal-affine structure of $G L(N)$ groups (in particular $G L(4)$ ) and, passing from a given metric to another, gives rise to induced scalar fields that, as we shall show below, can be generated by a dimensional reduction mechanism. As we shall show below, this feature can be considered as a sort of gravitational Higgs-like mechanism capable of inducing symmetry breakings.

\section{THE 5D-SPACE AND THE REDUCTION TO 4D-DYNAMICS}

\section{A. The 5D-formalism}

In this section, we are going to define the curvature invariants, the field equations and the conservation laws in the 5D-space. In general, we ask for a space which is a smooth manifold, singularity free and, first of all, defined in such a way that conservation laws on it are valid. Technically given, a 2-rank tensor of the form $T^{A B}$, the relation $\nabla_{A} T_{B}^{A}=0$ must be always valid and never singular in the sense that it is preserved by diffeomorphisms in any coordinate frame. The 5D-Riemann tensor is

$$
R_{A B C}^{D}=\partial_{B} \Gamma_{A C}^{D}-\partial_{C} \Gamma_{A B}^{D}+\Gamma_{E B}^{D} \Gamma_{A C}^{E}-\Gamma_{E C}^{D} \Gamma_{A B}^{E} .
$$

The number of independent components of such a tensor, after the full derivation and thanks to the Petrov classification [59], is $\frac{1}{12} N^{2}\left(N^{2}-1\right)=50$. The Ricci tensor and scalar are derived from the contractions

$$
R_{A B}=R_{A C B}^{C}, \quad{ }^{(5)} R=R_{A}^{A} .
$$

The field equations can be derived from the 5D-HilbertEinstein action 


$$
{ }^{(5)} \mathcal{A}=-\frac{1}{16 \pi^{(5)} G} \int d^{5} x \sqrt{-g^{(5)}}\left[{ }^{(5)} R\right] \text {, }
$$

where, using the standard notation, ${ }^{(5)} G$ is the $5 \mathrm{D}$ gravitational coupling constant and $g^{(5)}$ is the determinant of the 5D-metric. The variational principle

$$
\delta \int d^{5} x \sqrt{-g^{(5)}}\left[{ }^{(5)} R\right]=0,
$$

gives the 5D-field equations which are

$$
G_{A B}=R_{A B}-\frac{1}{2} g_{A B}{ }^{(5)} R=0,
$$

so that at least the Ricci-flat space is always a solution. Let us define now the 5D-stress-energy tensor:

$$
T_{A B}=\nabla_{A} \Phi \nabla_{B} \Phi-\frac{1}{2} g_{A B} \nabla_{C} \Phi \nabla^{C} \Phi,
$$

where only the kinetic terms are present. As standard, such a tensor can be derived from a variational principle

$$
T^{A B}=\frac{2}{\sqrt{-g^{(5)}}} \frac{\delta\left(\sqrt{-g^{(5)}} \mathcal{L}_{\Phi}\right)}{\delta g_{A B}},
$$

where $\mathcal{L}_{\Phi}$ is a Lagrangian density connected with the scalar field $\Phi$ whose physical meaning will be clear below. Because of the definition of 5D space itself [42], it is important to stress now that no self-interaction potential $V(\Phi)$ has been taken into account so that $T_{A B}$ is a completely symmetric object and $\Phi$ is, by definition, a cyclic variable. This fact guarantees that Noether theorem holds for $T_{A B}$ and a conservation law intrinsically exists. With these considerations in mind, the field equations can now assume the form

$$
R_{A B}=\chi\left(T_{A B}-\frac{1}{2} g_{A B} T\right),
$$

where $T$ is the trace of $T_{A B}$ and $\chi=8 \pi^{(5)} G$, being $\hbar=c=1$. The form (84) of field equations is useful in order to put in evidence the role of the scalar field $\Phi$, if we are not simply assuming Ricci-flat 5D-spaces. As we said, $T_{A B}$ is a symmetric tensor for which the relation

$$
T_{[A, B]}=T_{A B}-T_{B A}=0,
$$

holds. Due to the choice of the metric and to the symmetric nature of the stress-energy tensor $T_{A B}$ and of the Einstein field equations $G_{A B}$, the contracted Bianchi identities

$$
\nabla_{A} T_{B}^{A}=0, \quad \nabla_{A} G_{B}^{A}=0,
$$

hold. Developing the stress-energy tensor, we have

$$
\begin{aligned}
\nabla_{A} T_{B}^{A}= & \nabla_{A}\left(\partial_{B} \Phi \partial^{A} \Phi-\frac{1}{2} \delta_{B}^{A} \partial_{C} \Phi \partial^{C} \Phi\right)= \\
= & \left(\nabla_{A} \Phi_{B}\right) \Phi^{A}+\Phi_{B}\left(\nabla_{A} \Phi^{A}\right)- \\
& +\frac{1}{2}\left(\nabla_{B} \Phi_{C}\right) \Phi^{C}-\frac{1}{2} \Phi_{C}\left(\nabla_{B} \Phi^{C}\right)= \\
= & \left(\nabla_{A} \Phi_{B}\right) \Phi^{A}+\Phi_{B}\left(\nabla_{A} \Phi^{A}\right)-\Phi_{C}\left(\nabla_{B} \Phi^{C}\right)
\end{aligned}
$$

Since our 5D-space is a Riemannian manifold, it is

$$
\nabla_{A} \Phi_{B}=\nabla_{B} \Phi_{A}
$$

and then

$$
\begin{aligned}
& \Phi^{A}\left(\nabla_{A} \Phi_{B}\right)-\Phi_{C}\left(\nabla_{B} \Phi^{C}\right)= \\
& =\Phi^{A}\left(\nabla_{B} \Phi_{A}\right)-\Phi_{C}\left(\nabla_{B} \Phi^{C}\right)=0 .
\end{aligned}
$$

In this case, partial and covariant derivatives coincide for the scalar field $\Phi$. Finally

$$
\nabla_{A} T_{B}^{A}=\Phi_{B}^{(5)} \square \Phi
$$

where ${ }^{(5)} \square$ is the $5 \mathrm{D}$ d'Alembert operator defined as $\nabla_{A} \Phi^{A} \equiv g^{A B} \Phi_{, A ; B} \equiv{ }^{(5)} \square \Phi$. The general result is that the conservation of the stress-energy tensor $T_{A B}$ (i.e. the contracted Bianchi identities) implies the Klein-Gordon equation which assigns the dynamics of $\Phi$, that is

$$
\nabla_{A} T_{A}^{B}=0 \quad \Longleftrightarrow \quad{ }^{(5)} \square \Phi=0,
$$

assuming $\Phi_{B} \neq 0$ since we are dealing with a non-trivial physical field. Let us note again the absence of selfinteraction (i.e. potential) terms. As we shall see below, the relation (91), being a field equation, gives a physical meaning to the fifth dimension.

The reduction to the $4 \mathrm{D}$-dynamics can accomplished by taking into account the Campbell theorem [60]. This theorem states that it is always possible to consider a $4 \mathrm{D}$ Riemannian manifold, defined by the line element $d s^{2}=$ $g_{\alpha \beta} d x^{\alpha} d x^{\beta}$, in a 5D one with $d S^{2}=g_{A B} d x^{A} d x^{B}$.

We have $g_{A B}=g_{A B}\left(x^{\alpha}, x^{4}\right)$ with $x^{4}$ the yet unspecified extra coordinate. As we discussed above, $g_{A B}$ is covariant under the group of $5 \mathrm{D}$ coordinate transformations $x^{A} \rightarrow \bar{x}^{A}\left(x^{B}\right)$, but not under the (restricted) group of $4 \mathrm{D}$ transformations $x^{\alpha} \rightarrow \bar{x}^{\alpha}\left(x^{\beta}\right)$. This fact has, as a relevant consequence, that the choice of $5 \mathrm{D}$ coordinates results as the gauge necessary to specify the $4 \mathrm{D}$ physics also in its non-standard aspects. Vice-versa, in specifying the $4 \mathrm{D}$ physics, the bijective embedding process in $5 \mathrm{D}$ gives physical meaning to the fifth coordinate $x^{4}$. In other words, the fifth coordinate, in 4D can assume the physical meaning of the mass. 


\section{B. The reduction to $4 \mathrm{D}$}

Let us replace the variational principle (80) with

$$
\delta \int d^{(5)} x \sqrt{-g^{(5)}}\left[{ }^{(5)} R+\lambda\left(g_{44}-\varepsilon \Phi^{2}\right)\right]=0,
$$

where $\lambda$ is a Lagrange multiplier, $\Phi$ a scalar field and $\varepsilon= \pm 1$. This approach is completely general and used in theoretical physics when we want to put in evidence some specific feature [103]. In this case, we need it in order to derive the physical gauge for the $5 \mathrm{D}-$ metric. We can write down the metric as

$$
\begin{aligned}
d S^{2}= & g_{A B} d x^{A} d x^{B}=g_{\alpha \beta} d x^{\alpha} d x^{\beta}+g_{44}\left(d x^{4}\right)^{2} \\
& =g_{\alpha \beta} d x^{\alpha} d x^{\beta}+\varepsilon \Phi^{2}\left(d x^{4}\right)^{2}
\end{aligned}
$$

from which we obtain directly particle-like solutions $(\varepsilon=$ $-1)$ or wave-like solutions $(\varepsilon=+1)$ in the 4 D-reduction procedure. The standard signature of $4 \mathrm{D}$-component of the metric is $(+---)$ and $\alpha, \beta=0,1,2,3$. Furthermore, the 5D-metric can be written in a Kaluza-Klein fashion as the matrix

$$
g_{A B}=\left(\begin{array}{cc}
g_{\alpha \beta} & 0 \\
0 & \varepsilon \Phi^{2}
\end{array}\right)
$$

and the 5D-curvature Ricci tensor is

$$
\begin{aligned}
{ }^{(5)} R_{\alpha \beta}= & R_{\alpha \beta}-\frac{\Phi, \alpha ; \beta}{\Phi}+\frac{\varepsilon}{2 \Phi^{2}}\left(\frac{\Phi_{, 4} g_{\alpha \beta, 4}}{\Phi}-g_{\alpha \beta, 44}+\right. \\
& \left.+g^{\lambda \mu} g_{\alpha \lambda, 4} g_{\beta \mu, 4}-\frac{g^{\mu \nu} g_{\mu \nu, 4} g_{\alpha \beta, 4}}{2}\right)
\end{aligned}
$$

where $R_{\alpha \beta}$ is the $4 \mathrm{D}$-Ricci tensor. The expressions for ${ }^{(5)} R_{44}$ and ${ }^{(5)} R_{4 \alpha}$ can be analogously derived. After the projection from $5 \mathrm{D}$ to $4 \mathrm{D}, g_{\alpha \beta}$, derived from $g_{A B}$, no longer explicitly depends on $x^{4}$, so, from Eq.95), a useful expression for the Ricci scalar can be derived:

$$
{ }^{(5)} R=R-\frac{1}{\Phi} \square \Phi,
$$

where the dependence on $\varepsilon$ is explicitly disappeared and $\square$ is the 4D-d'Alembert operator which gives $\square \Phi \equiv$ $g^{\mu \nu} \Phi_{, \mu ; \nu}$. The action in Eq. (92) can be recast in a $4 \mathrm{D}-$ reduced Brans-Dicke action of the form

$$
\mathcal{A}=-\frac{1}{16 \pi G_{N}} \int d^{4} x \sqrt{-g}\left[\Phi R+\mathcal{L}_{\Phi}\right],
$$

where the Newton constant is given by

$$
G_{N}=\frac{{ }^{(5)} G}{2 \pi l}
$$

with $l$ a characteristic length in $5 \mathrm{D}$ which can be related to a suitable Compton length. Defining a generic function of a 4D-scalar field $\phi$ as

$$
-\frac{\Phi}{16 \pi G_{N}}=F(\phi)
$$

we get, in $4 \mathrm{D}$, a general action in which gravity is nonminimally coupled to a scalar field, that is

$$
\begin{aligned}
\mathcal{A}= & \int_{\mathcal{M}} d^{4} x \sqrt{-g}\left[F(\phi) R+\frac{1}{2} g^{\mu \nu} \phi_{; \mu} \phi_{; \nu}-V(\phi)\right]+ \\
& +\int_{\partial \mathcal{M}} d^{3} x \sqrt{-b} K
\end{aligned}
$$

where the form and the role of $V(\phi)$ are still general. The second integral is a boundary term where $K \equiv h^{i j} K_{i j}$ is the trace of the extrinsic curvature tensor $K_{i j}$ of the hypersurface $\partial \mathcal{M}$ which is embedded in the $4 \mathrm{D}$-manifold $\mathcal{M} ; b$ is the metric determinant of the 3D-manifold.

The Einstein field equations can be derived by varying with respect to the $4 \mathrm{D}$-metric $g_{\mu \nu}$

$$
G_{\mu \nu}=R_{\mu \nu}-\frac{1}{2} g_{\mu \nu} R=\tilde{T}_{\mu \nu},
$$

where

$$
\begin{aligned}
\tilde{T}_{\mu \nu}= & \frac{1}{F(\phi)}\left\{-\frac{1}{2} \phi_{; \mu} \phi_{; \nu}+\frac{1}{4} g_{\mu \nu} \phi_{; \alpha} \phi^{; \alpha}+\right. \\
& \left.-\frac{1}{2} g_{\mu \nu} V(\phi)-g_{\mu \nu} \square F(\phi)+F(\phi)_{; \mu \nu}\right\}
\end{aligned}
$$

is the effective stress-energy tensor containing the nonminimal coupling contributions, the kinetic terms and the potential of the scalar field $\phi$. In the case in which $F(\phi)$ is a constant $F_{0}$ (in our units, $F_{0}=-1 /\left(16 \pi G_{N}\right)$ ), we get the stress-energy tensor of a scalar field minimally coupled to gravity, that is

$$
T_{\mu \nu}=\phi_{; \mu} \phi_{; \nu}-\frac{1}{2} g_{\mu \nu} \phi_{; \alpha} \phi^{; \alpha}+g_{\mu \nu} V(\phi) .
$$

By varying with respect to $\phi$, we get the 4D-KleinGordon equation

$$
\square \phi-R F^{\prime}(\phi)+V^{\prime}(\phi)=0,
$$

where $F^{\prime}(\phi)=d F(\phi) / d \phi$ and $V^{\prime}(\phi)=d V(\phi) / d \phi$. It is possible to show that Eq. (104) is nothing else but the contracted Bianchi identity. This feature shows that the effective stress-energy tensor at right hand side of (101) is a zero-divergence tensor and this fact is fully compatible with Einstein theory of gravity also if we started from a 5D-space. Specifically, the reduction procedure, which we have used, preserves the standard features of GR since we are in the realm of the conformal-affine structure discussed above.

In order to physically identify the fifth dimension, let us recast the above Klein-Gordon equation (104) as

$$
\left(\square+m_{\text {eff }}^{2}\right) \phi=0,
$$

where

$$
m_{e f f}^{2}=\left[V^{\prime}(\phi)-R F^{\prime}(\phi)\right] \phi^{-1},
$$

is the effective mass, i.e. a function of $\phi$, where self-gravity contributions, $R F^{\prime}(\phi)$, and scalar field selfinteractions, $V^{\prime}(\phi)$, are taken into account. In any quantum field theory formulated on curved space-times, these 
contributions, at one-loop level, have the same "weight" [104. We want to show that a "natural" way to generate particle masses can be achieved starting from a $5 \mathrm{D}$ picture. In other words, the concept of mass can be derived from a geometric viewpoint.

\section{EXTENDED THEORIES OF GRAVITY}

\section{A. Effective gravity in $4 \mathrm{D}$}

The above scalar-tensor action for gravitational field is a particular case of a general class of effective theories in four dimensions which can be achieved by the reduction procedure. In general, starting from any higherdimensional theory, we can derive the class of effective actions [49, 50]

$$
\begin{aligned}
\mathcal{A}= & \int d^{4} x \sqrt{-g}\left[F\left(R, \square R, \square^{2} R, . . \square^{k} R, \phi\right)+\right. \\
& \left.-\frac{\varepsilon}{2} g^{\mu \nu} \phi_{; \mu} \phi_{; \nu}+\mathcal{L}_{m}\right]
\end{aligned}
$$

where $F$ is a generic function of curvature invariant 2 and scalar field $\phi$. It is interesting to point out that this theory looks similar to non-local gravities of general sort introduced in 51] and [52]. The term $\mathcal{L}_{m}$, as above, is the minimally coupled ordinary matter contribution. We shall use physical units $8 \pi G=c=\hbar=1$; $\varepsilon$ is a constant which specifies the theory. Actually its values can be $\varepsilon= \pm 1,0$ fixing the nature and the dynamics of the scalar field which can be a standard scalar field, a phantom field or a field without dynamics [63, 64] .

In the metric approach, the field equations are obtained by varying (107) with respect to $g_{\mu \nu}$. We get

$$
\begin{aligned}
G^{\mu \nu}= & \frac{1}{\mathcal{G}}\left[T^{\mu \nu}+\frac{1}{2} g^{\mu \nu}(F-\mathcal{G} R)+\left(g^{\mu \lambda} g^{\nu \sigma}-\right.\right. \\
& \left.+g^{\mu \nu} g^{\lambda \sigma}\right) \mathcal{G}_{; \lambda \sigma}+\frac{1}{2} \sum_{i=1}^{k} \sum_{j=1}^{i}\left(g^{\mu \nu} g^{\lambda \sigma}+\right. \\
& \left.+g^{\mu \lambda} g^{\nu \sigma}\right)\left(\square^{j-i}\right)_{; \sigma}\left(\square^{i-j} \frac{\partial F}{\partial \square^{i} R}\right)_{; \lambda}- \\
& \left.g^{\mu \nu} g^{\lambda \sigma}\left(\left(\square^{j-1} R\right)_{; \sigma} \square^{i-j} \frac{\partial F}{\partial \square^{i} R}\right)_{; \lambda}\right]
\end{aligned}
$$

where $G^{\mu \nu}$ is the above Einstein tensor and

$$
\mathcal{G} \equiv \sum_{j=0}^{n} \square^{j}\left(\frac{\partial F}{\partial \square^{j} R}\right)
$$

is a scalar function which determines the coupling.

\footnotetext{
${ }^{2}$ Other curvature invariants like $R_{\mu \nu} R^{\mu \nu}, R_{\mu \nu \alpha \beta} R^{\mu \nu \alpha \beta}$, $C_{\mu \nu \alpha \beta} C^{\mu \nu \alpha \beta}$ are also possible, as we will show below.
}

The differential Eqs.(108) are of order $(2 k+4)$. The stress-energy tensor is due to the kinetic part of the scalar field and to the ordinary matter:

$$
T_{\mu \nu}=T_{\mu \nu}^{m}+\frac{\varepsilon}{2}\left[\phi_{; \mu} \phi_{; \nu}-\frac{1}{2} \phi^{; \alpha} \phi_{; \alpha}\right] .
$$

The (possible) contribution of a potential $V(\phi)$ is contained in the definition of $F$. From now on, we shall indicate by a capital $F$ a Lagrangian density containing also the contribution of a potential $V(\phi)$ and by $F(\phi)$, $f(R)$, or $f(R, \square R)$ a function of such fields without potential.

By varying with respect to the scalar field $\phi$, we obtain the Klein-Gordon equation

$$
\varepsilon \square \phi=-\frac{\partial F}{\partial \phi} .
$$

The simplest class of (107) theories extending GR is achieved assuming

$$
F=f(R), \quad \varepsilon=0,
$$

in the action (107); $f(R)$ is an arbitrary (analytic) function of the Ricci curvature scalar $R$. The standard Hilbert-Einstein action is recovered for $f(R)=R$. Varying with respect to $g_{\alpha \beta}$, we get the field equations

$$
f^{\prime}(R) R_{\alpha \beta}-\frac{1}{2} f(R) g_{\alpha \beta}=f^{\prime}(R)^{;^{\mu \nu}}\left(g_{\alpha \mu} g_{\beta \nu}-g_{\alpha \beta} g_{\mu \nu}\right)
$$

which are fourth-order equations due to the term $f^{\prime}(R)^{; \mu \nu}$; the prime indicates the derivative with respect to $R$. Eq. (113) is also the equation for $T_{\mu \nu}=0$ when the matter term is absent.

By a suitable manipulation, the above equation can be rewritten as:

$$
\begin{aligned}
G_{\alpha \beta}= & \frac{1}{f^{\prime}(R)}\left\{\frac{1}{2} g_{\alpha \beta}\left[f(R)-R f^{\prime}(R)\right]+f^{\prime}(R)_{; \alpha \beta}-\right. \\
& \left.+g_{\alpha \beta} \square f^{\prime}(R)\right\}+\frac{T_{\alpha \beta}^{m}}{f^{\prime}(R)}=T_{\alpha \beta}^{c u r v}+\frac{T_{\alpha \beta}^{m}}{f^{\prime}(R)},
\end{aligned}
$$

where $T_{\alpha \beta}^{c u r v}$ is an effective stress-energy tensor constructed by the extra curvature terms and standard matter contribution has been also considered. In the case

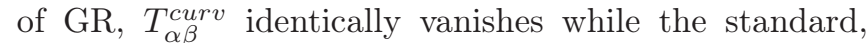
minimal coupling is recovered for the matter contribution. The peculiar behavior of GR, that is $f(R)=R$, is due to the particular form of the Lagrangian itself which, even though it is a second order Lagrangian, can be non-covariantly rewritten as the sum of a first order Lagrangian plus a pure divergence term. The HilbertEinstein Lagrangian can be recast as follows:

$$
\begin{aligned}
\mathcal{L}_{H E}= & \sqrt{-g}\left[p^{\alpha \beta}\left(\Gamma_{\alpha \sigma}^{\rho} \Gamma_{\rho \beta}^{\sigma}-\Gamma_{\rho \sigma}^{\rho} \Gamma_{\alpha \beta}^{\sigma}\right)+\right. \\
& \left.+\nabla_{\sigma}\left(p^{\alpha \beta} u^{\sigma}{ }_{\alpha \beta}\right)\right]
\end{aligned}
$$


where:

$$
p^{\alpha \beta}=\sqrt{-g} g^{\alpha \beta}=\frac{\partial \mathcal{L}_{H E}}{\partial R_{\alpha \beta}},
$$

$\Gamma$ is the Levi-Civita connection of $g$ and $u_{\alpha \beta}^{\sigma}$ is a quantity constructed from the variation of $\Gamma$ [59]. Since $u_{\alpha \beta}^{\sigma}$ is not a tensor, the above expression is not covariant; however a standard procedure has been studied to recast covariance in the first order theories [65]. This clearly shows that the field equations should consequently be second order and the Hilbert-Einstein Lagrangian is thus degenerate. In other words, we can say that the only degenerate theory of gravity (with respect to the class of $f(R)$-gravity) is GR being its Hessian determinant null.

From the action (107), it is possible to obtain the case discussed in the previous section by choosing

$$
F=F(\phi) R-V(\phi), \quad \varepsilon=-1,
$$

and then

$$
\mathcal{A}=\int d^{4} x \sqrt{-g}\left[F(\phi) R+\frac{1}{2} g^{\mu \nu} \phi_{; \mu} \phi_{; \nu}-V(\phi)\right] .
$$

Several other interesting cases can be obtained by suitable choices of the function $F$. However, all these models can be related by conformal-affine transformations.

\section{B. Conformal Transformations}

It is possible to show that any higher-order or scalartensor theory, in absence of ordinary matter, e.g. a perfect fluid, is conformally equivalent to an Einstein theory plus minimally coupled scalar fields (see [49, 50] for details). If standard matter is present, conformal transformations allow to transfer non-minimal coupling to the matter component [66]. The conformal transformation on the metric $g_{\mu \nu}$ is

$$
\tilde{g}_{\mu \nu}=e^{2 \omega} g_{\mu \nu},
$$

where $e^{2 \omega}$ is the conformal factor. Under this transformation, the Lagrangian in (118) becomes

$$
\begin{aligned}
& \sqrt{-g}\left(F R+\frac{1}{2} g^{\mu \nu} \phi_{; \mu} \phi_{; \nu}-V\right)=\sqrt{-\tilde{g}} e^{-2 \omega}(F \tilde{R}- \\
& \left.+6 F \square_{\tilde{g}} \omega-6 F \omega_{; \alpha} \omega^{; \alpha}+\frac{1}{2} \tilde{g}^{\mu \nu} \phi_{; \mu} \phi_{; \nu}-e^{-2 \omega} V\right),
\end{aligned}
$$

in which $\tilde{R}$ and $\square_{\tilde{g}}$ are the Ricci scalar and the d'Alembert operator relative to the metric $\tilde{g}$. Requiring the theory in the metric $\tilde{g}_{\mu \nu}$ to appear as a standard Einstein theory [107], the conformal factor has to be related to $F$, that is

$$
e^{2 \omega}=-2 F \text {. }
$$

where $F$ must be negative in order to restore physical coupling in our adopted signature. Using this relation and introducing a new scalar field $\tilde{\phi}$ and a new potential $\tilde{V}$, defined respectively by

$$
\tilde{\phi}_{; \alpha}=\sqrt{\frac{3 F_{\phi}^{2}-F}{2 F^{2}}} \phi_{; \alpha}, \quad \tilde{V}(\tilde{\phi}(\phi))=\frac{V(\phi)}{4 F^{2}(\phi)},
$$

the Lagrangian (120) becomes

$$
\begin{aligned}
& \sqrt{-g}\left(F R+\frac{1}{2} g^{\mu \nu} \phi_{; \mu} \phi_{; \nu}-V\right)= \\
& \quad=\sqrt{-\tilde{g}}\left(-\frac{1}{2} \tilde{R}+\frac{1}{2} \tilde{\phi}_{; \alpha} \tilde{\phi}^{; \alpha}-\tilde{V}\right),
\end{aligned}
$$

which is the standard Hilbert-Einstein Lagrangian plus the Lagrangian of a minimally coupled scalar field $\tilde{\phi}$. Therefore, every non-minimally coupled scalar-tensor theory, in absence of ordinary matter, e.g. perfect fluid, is conformally equivalent to an Einstein theory, being the conformal transformation and the potential suitably defined by (121) and (122). The converse is also true: for a given $F(\phi)$, such that $3 F_{\phi}^{2}-F>0$, we can transform a standard Einstein theory into a non-minimally coupled scalar-tensor theory. This means that, in principle, if we are able to solve the field equations in the framework of the Einstein theory in presence of a scalar field with a given potential, we should be able to get the solutions for the scalar-tensor theories, assigned by the coupling $F(\phi)$, via the conformal transformation (121) with the constraints given by (122). Following the standard terminology, the "Einstein frame" is the framework of the Einstein theory with the minimal coupling and the "Jordan frame" is the framework of the non-minimally coupled theory [66].

Performing the conformal transformation (119) in the case of $f(R)$-gravity, the field equations (114) become:

$$
\begin{aligned}
\tilde{G}_{\alpha \beta}= & \frac{1}{f^{\prime}(R)}\left\{\frac{1}{2} g_{\alpha \beta}\left[f(R)-R f^{\prime}(R)\right]+f^{\prime}(R)_{; \alpha \beta}-\right. \\
& \left.+g_{\alpha \beta} \square f^{\prime}(R)\right\}+2\left(\omega_{; \alpha ; \beta}+g_{\alpha \beta} \square \omega-\omega_{; \alpha} \omega_{; \beta}+\right. \\
& \left.+\frac{1}{2} g_{\alpha \beta} \omega_{; \gamma} \omega^{; \gamma}\right) .
\end{aligned}
$$

The conformal factor is

$$
\omega=\frac{1}{2} \ln \left|f^{\prime}(R)\right|,
$$

which has to be substituted into (114). Rescaling $\omega$ in such a way that

$$
k \phi=\omega,
$$

and $k=\sqrt{1 / 6}$, we obtain the Lagrangian equivalence

$$
\sqrt{-g} f(R)=\sqrt{-\tilde{g}}\left(-\frac{1}{2} \tilde{R}+\frac{1}{2} \phi_{; \alpha} \phi^{; \alpha}-\tilde{V}\right),
$$


and the Einstein equations in standard form

$$
\tilde{G}_{\alpha \beta}=\phi_{; \alpha} \phi_{; \beta}-\frac{1}{2} \tilde{g}_{\alpha \beta} \phi_{; \gamma} \phi^{; \gamma}+\tilde{g}_{\alpha \beta} V(\phi),
$$

with the potential

$$
\begin{aligned}
V(\phi)= & \frac{e^{-4 k \phi}}{2}\left[\mathcal{P}(\phi)-\mathcal{N}\left(e^{2 k \phi}\right) e^{2 k \phi}\right]= \\
= & \frac{1}{2} \frac{f(R)-R f^{\prime}(R)}{f^{\prime}(R)^{2}} .
\end{aligned}
$$

Here $\mathcal{N}$ is the inverse function of $\mathcal{P}^{\prime}(\phi)$ and $\mathcal{P}(\phi)=$ $\int \exp (2 k \phi) d \mathcal{N}$. However, the problem is completely solved if $\mathcal{P}^{\prime}(\phi)$ can be analytically inverted. In summary, a fourth-order theory is conformally equivalent to the standard second-order Einstein theory plus a scalar field.

This procedure can be always extended to more general theories. If the theory is assumed to be higher than fourth order, we may have Lagrangian densities of the form,

$$
\mathcal{L}=\mathcal{L}\left(R, \square R, \ldots \square^{k} R\right) .
$$

As we have seen, any $\square$ operator introduces two further terms of derivation into the field equations. For example a theory like

$$
\mathcal{L}=R \square R,
$$

is a sixth-order theory and the above approach can be pursued by considering a conformal factor of the form

$$
\omega=\frac{1}{2} \ln \left|\frac{\partial \mathcal{L}}{\partial R}+\square \frac{\partial \mathcal{L}}{\partial \square R}\right| .
$$

In general, increasing two orders of derivation in the field equations (i.e. for every term $\square R$ ), corresponds to adding a scalar field in the conformally transformed frame. A sixth-order theory can be reduced to an Einstein theory plus two minimally coupled scalar fields; a $2 n$-order theory can be, in principle, reduced to an Einstein theory plus $(n-1)$-scalar fields. On the other hand, these considerations can be directly generalized to higher-order-scalar-tensor theories in any number of dimensions. Due to the conformal transformations (and the conformal-affine invariance), the physical information remains the same. This means that any two further degrees of freedom can be recast as a scalar field (of gravitational origin) whose dynamics is given by an effective Klein-Gordon equation. We want to show that such further gravitational degrees of freedom can play a fundamental role inducing spontaneous symmetry breaking and then generating the mass of observed particles.

\section{THE GENERATION OF MASSES}

\section{A. Particle masses as eigenstates from 5D}

The above considerations allow a straightforward mechanism for the generation of masses that can be related to the projection from $5 \mathrm{D}$ to $4 \mathrm{D}$-manifolds. Let us start with a toy model that can immediately illustrate the mechanism. In a flat 5D-space-time, the 5D d'Alembert operator can be split, following the metric definition (93) for particle-like solutions, as:

$$
{ }^{(5)} \square=\square-\partial_{4}{ }^{2},
$$

selecting the value $\varepsilon=-1$ in the metric. Introducing the scalar field $\Phi$, generated by the projection through the Lagrange multiplier in the action (80), we have

$$
{ }^{(5)} \square \Phi=\left[\square-\partial_{4}{ }^{2}\right] \Phi=0,
$$

and then

$$
\square \Phi=\partial_{4}{ }^{2} \Phi .
$$

The problem is solvable by separation of variables since the metric matrix (94) is diagonal in the fifth component. We split the scalar field $\Phi$ into two functions

$$
\Phi=\phi(t, \vec{x}) \psi\left(x_{4}\right),
$$

where the field $\phi$ depends on the ordinary space-time coordinates, while $\chi$ is a function of the fifth coordinate $x_{4}$. Inserting (135) into Eq. (134), we get

$$
\frac{\square \phi}{\phi}=\frac{1}{\psi}\left[\frac{d^{2} \psi}{d x_{4}^{2}}\right]=-k_{n}^{2},
$$

where $k_{n}$ must be a constant for consistency. From Eq.(136), we obtain the two equations of motion

$$
\left(\square+k_{n}^{2}\right) \phi=0,
$$

and

$$
\frac{d^{2} \psi}{d x_{4}^{2}}+k_{n}^{2} \psi=0
$$

Eq. (137) is the evolution equation for the further gravitational degrees of freedom that we will discuss below. Eq.(138) describes a harmonic oscillator whose general solution is

$$
\psi\left(x_{4}\right)=c_{1} e^{-i k_{n} x_{4}}+c_{2} e^{i k_{n} x_{4}} .
$$

The constant $k_{n}$ has the physical dimension of the inverse of a length and, assigning boundary conditions, we can derive the eigenvalue relation

$$
k_{n}=\frac{2 \pi}{l} n,
$$

where $n$ is an integer and $l$ a length which we have previously defined in Eq. (98). As a result, in standard units, we recover the Compton length

$$
\lambda_{n}=\frac{\hbar}{2 \pi m_{n} c}=\frac{1}{k_{n}},
$$


which assigns the mass of a particle. It has to be stressed that, the eigenvalues of Eq. (138) are the masses of particles which are generated by the reduction process from $5 \mathrm{D}$ to $4 \mathrm{D}$. On the other hand, different values of $n$ fix the families of particles, while, for any given value of $n$, the parameters $c_{1,2}$ distinguish a particle and an antiparticle within a family. This toy model can be refined by considering other quantum numbers of particles. For the moment, we are interested only in the problem of mass generation.

\section{B. 4D-dynamics of the scalar field $\Phi$}

Dynamics of 4D-component of the induced scalar field $\Phi$, see Eq. (135), can be related to the space-time deformations discussed above. In this way, the role of $G L(4)-$ group of diffeomorphism will result of fundamental importance. Let us start our consideration by showing how particles can acquire mass by deformations and let us relate the procedure with the above reduction mechanism. As standard, a particle with zero mass is characterized by the invariant relation

$$
\eta^{\alpha \beta} p_{\alpha} p_{\beta}=0,
$$

in the Minkowski space. Deforming the space-time and considering the above operators, one has

$$
\eta^{A B} \Phi_{A}^{C} \Phi_{B}^{D} e_{C}^{\alpha} e_{D}^{\beta} p_{\alpha} p_{\beta}=g^{\alpha \beta} p_{\alpha} p_{\beta}=0,
$$

so we have defined two frames, one, the Minkowski spacetime, defined by the metric $\eta^{\alpha \beta}$ and the other defined by the metric $g^{\alpha \beta}$, generated by the projection from $5 \mathrm{D}$. The two frames are related by the matrices of deformation functions $\Phi_{A}^{C}(x)$. In both frames the massless particle follow a null path, but we observe that using the decomposition (69) the particle does not appear massless with respect to the first frame. As matter of fact, Eq. (143) becomes

$$
\Omega^{-2} \eta^{\alpha \beta} p_{\alpha} p_{\beta}+\chi^{\alpha \beta} p_{\alpha} p_{\beta}=0
$$

so that

$$
\eta^{\alpha \beta} p_{\alpha} p_{\beta}=-\Omega^{2} \chi^{\alpha \beta} p_{\alpha} p_{\beta} \neq 0 .
$$

and now in the first frame we are able to define a rest reference system for the particle.

For a massless particle it is not possible to define a rest reference system since considering the invariant relation,

$$
g^{00} p_{0}^{2}+2 g^{0 i} p_{0} p_{i}+g^{i j} p_{i} p_{j}=0,
$$

and defining as rest frame the system in which $p_{j}=0$, the solution exists only for $p_{0}=0$ i.e. only the trivial solution $p_{\alpha}=0$ satisfies the " rest reference frame" condition.

On the other hand, if we consider massive particle, then

$$
g^{00} p_{0}^{2}+2 g^{0 i} p_{0} p_{i}+g^{i j} p_{i} p_{j}=m^{2},
$$

the rest frame is characterized by the conditions $p_{j}=$ 0 and consequently $g^{00} p_{0}^{2}=m^{2}$. This means that the (squared) mass is proportional to the (squared) energy and the solution is no more trivial.

To overcome this problem, let us fix the deformation such that

$$
-\Omega^{2} \chi^{\alpha \beta} p_{\alpha} p_{\beta}=m^{2},
$$

$m$ being the mass "attribute" to the particle.

In the second frame we have

$$
p_{0}^{2}-\vec{p}^{2}+\Omega^{2} \chi^{00} p_{0}^{2}+2 \chi^{0 i} p_{0} p_{i}+\chi^{i j} p_{i} p_{j}=0,
$$

if $\vec{p}=0$ then

$$
p_{0}^{2}\left(1+\Omega^{2} \chi^{00}\right)=1
$$

which implies, besides the trivial solution, also the condition $\Omega^{2} \chi^{00}=-1$ which, together with Eq. (148), gives

$$
p_{0}^{2}=m^{2},
$$

the rest system condition in the first frame.

It is also possible to show that there is an equivalence between deforming a metric and giving mass to a massless particle. As we have shown, we have described the spacetime deformation by using matrices of scalar fields. The same problem can be addressed in terms of space-time tensors

$$
\eta_{A B} \Phi_{C}^{A} \Phi_{D}^{B} e_{\mu}^{C} e_{\nu}^{D}=g_{\alpha \beta} \Phi_{\mu}^{\alpha} \Phi_{\nu}^{\beta}=\tilde{g}_{\mu \nu},
$$

where we have used the tetrad

$$
e_{A}^{\mu} e_{\mu}^{B}=\delta_{A}^{B} .
$$

It should be observed that the $\Phi_{\mu}^{\alpha}$ do not represent coordinates transformations as far as they cannot be reduced to Jacobian matrices. This means that $\Phi_{\mu}^{\alpha}$ have a fundamental physical meaning. Starting from $\tilde{g}$ we observe that if $g_{\mu \nu} p^{\mu} p^{\nu}=0$ then $\tilde{g}_{\mu \nu} p^{\mu} p^{\nu} \neq 0$ when $\tilde{g}$ is a general deformation that can be related to the conformal transformations of $g$. Eq. (152) tells us that, equivalently, we can read the deformation as a transformation of the 4-momentum of the particle

$$
\tilde{g}_{\mu \nu} p^{\mu} p^{\nu}=g_{\alpha \beta} \Phi_{\mu}^{\alpha} \Phi_{\nu}^{\beta} p^{\mu} p^{\nu} \equiv g_{\alpha \beta} \tilde{p}^{\alpha} \tilde{p}^{\beta},
$$

in such a way there exists deformations of space-time which give mass to massless particles. In other words, deformations, that are elements of the conformallyinvariant $G L(4)$-group can be related, in principle, to the generation of the masses of particles.

\section{A Lagrangian Approach for mass generation from deformations}

So far we have considered the geometrical definition of mass from the point of view of relativistic mechanics. 
Now we would like to extend this result to classical field theory with the aim to extend it to quantum field theory.

Let us consider a scalar free massless particle. It can be described by the Lagrangian

$$
\begin{aligned}
\mathcal{L}= & \frac{1}{2} \sqrt{-g} g^{\mu \nu}\left(\partial_{\mu} \phi\right)\left(\partial_{\nu} \phi\right)= \\
& =\frac{1}{2} \sqrt{-g}\left(\eta^{\mu \nu}+\chi^{\mu \nu}\right)\left(\partial_{\mu} \phi\right)\left(\partial_{\nu} \phi\right) .
\end{aligned}
$$

It is well-known that the free propagator has a pole in $p^{2}=0$. In order to introduce a mass term in the Lagrangian and in the field equations, we have to eliminate a divergence from it, that is considering

$$
\begin{aligned}
& \partial_{\mu}\left[\partial_{\nu} \sqrt{-g} \chi^{\mu \nu} \phi^{2}\right]=\partial_{\mu} \partial_{\nu}\left(\sqrt{-g} \chi^{\mu \nu}\right) \phi^{2}+ \\
& +4 \partial_{\nu}\left(\sqrt{-g} \chi^{\mu \nu}\right) \phi \partial_{\mu} \phi+2 \sqrt{-g} \chi^{\mu \nu} \partial_{\mu} \phi \partial_{\nu} \phi+ \\
& +2 \sqrt{-g} \chi^{\mu \nu} \phi \partial_{\mu} \partial_{\nu} \phi
\end{aligned}
$$

the Lagrangian takes the form

$$
\begin{aligned}
\widetilde{\mathcal{L}}= & \frac{1}{2} \sqrt{-g}\left(\eta^{\mu \nu}\right)\left(\partial_{\mu} \phi\right)\left(\partial_{\nu} \phi\right)-\frac{1}{4} \partial_{\mu} \partial_{\nu}\left(\sqrt{-g} \chi^{\mu \nu}\right) \phi^{2}- \\
& +\partial_{\nu}\left(\sqrt{-g} \chi^{\mu \nu}\right) \phi\left(\partial_{\mu} \phi\right)-\frac{1}{2} \sqrt{-g} \chi^{\mu \nu} \phi \partial_{\mu} \partial_{\nu} \phi .
\end{aligned}
$$

In this way a "mass" term $m^{2}=\frac{1}{2} \partial_{\mu} \partial_{\nu}\left(\sqrt{-g} \chi^{\mu \nu}\right)$ can be defined in a new Lagrangian. On the other hand, considering an action

$$
\mathcal{A}=\int \sqrt{-g} \widetilde{\mathcal{L}}
$$

and a variational principle

$$
\frac{\delta \mathcal{A}}{\delta \phi}=0
$$

implies the equation

$$
\frac{\partial \widetilde{\mathcal{L}}}{\partial \phi}-\partial_{\alpha} \frac{\partial \widetilde{\mathcal{L}}}{\partial\left(\partial_{\alpha} \phi\right)}+\partial_{\alpha} \partial_{\beta} \frac{\partial \widetilde{\mathcal{L}}}{\partial\left(\partial_{\alpha} \partial_{\beta} \phi\right)}=0
$$

which gives

$$
\partial_{\mu}\left(\sqrt{-g} g^{\mu \nu} \partial_{\nu} \phi\right)=0
$$

or

$$
\square \phi=\eta^{\mu \nu} \partial_{\mu} \partial_{\nu} \phi+\Omega^{2} \chi^{\mu \nu} \partial_{\mu} \partial_{\nu} \phi=0 .
$$

It is well-known that this equation cannot give, in general, a potential or a mass term, except when we take as a solution a plane wave $\phi=\exp i k_{\mu} x^{\mu}$. In this case the $\chi$ part of the equation can be interpreted as a mass term according to

$$
\chi^{\mu \nu} \partial_{\mu} \partial_{\nu} \phi=-\Omega^{2} \chi^{\mu \nu} k_{\mu} k_{\nu} \exp i k_{\mu} x^{\mu}=m^{2} \phi .
$$

This equation can be compared with Eq. (148), previously derived for a relativistic particle. We can extend this result to each function which can be expressed by a Fourier transform,

$$
\phi(x)=\int \exp \left(i k_{\mu} x^{\mu}\right) \tilde{f}(k) d^{4} k,
$$

where the space-time dependent mass term is defined by the equation

$$
m^{2}(x)=-\int \chi^{\mu \nu} k_{\mu} k_{\nu} \exp \left(i k_{\mu} x^{\mu}\right) \tilde{f}(k) d^{4} k .
$$

With these restrictions, the Klein-Gordon equation for a massless particle in the $g$-frame is seen in the $\eta$-frame as a massive particle as soon as the plane wave solutions are considered. This result makes more sense when the mass is interpreted as a quantum effect.

In the case a curved space-time, the above arguments can be implemented by the substitutions of operators

$$
\begin{aligned}
& \eta \rightarrow g \\
& \partial \rightarrow \nabla
\end{aligned}
$$

It is important to note that the scalar field equation is not conformally invariant. In order to have a conformally invariant scalar field equation, it is necessary to introduce, in the Lagrangian density a non-minimal coupling between geometry and field. A possible choice is

$$
\mathcal{L}=\frac{1}{2} \sqrt{-g} g^{\mu \nu}\left(\partial_{\mu} \phi\right)\left(\partial_{\nu} \phi\right)-\xi R(x) \phi^{2},
$$

that perfectly fits requirements of action (107) with suitable choices . By varying with respect to $\phi$, the KleinGordon equation

$$
\square \phi+\xi R \phi=0,
$$

is recovered. Minimal coupling is obtained for $\xi=0$. Conformal coupling is recovered for

$$
\xi=\frac{1}{4}[(N-2)(N-1)],
$$

in an $N$ dimensional space-time [104]. By analogy with the previous considerations, a deformation with constant curvature $R=m^{2}$ gives a mass term in the original frame. However, we need also to interpret the other terms appearing in the new frame. Expanding Eq. (167),

$\eta^{\mu \nu} \partial_{\mu} \partial_{\nu} \phi+\chi^{\mu \nu} \partial_{\mu} \partial_{\nu} \phi+\left(\eta^{\mu \nu}+\chi^{\mu \nu}\right) \Gamma_{\mu \nu}^{\lambda} \partial_{\lambda} \phi+\xi R \phi=0$

we need that the connection is compatible with the metric tensor $\left(\eta^{\mu \nu}+\chi^{\mu \nu}\right)$, that is

$$
\nabla\left(\eta^{\mu \nu}+\chi^{\mu \nu}\right)=0
$$

In order to have a deformation defining a mass $m^{2}=R$ in the original frame, two conditions must be satisfied,

$$
R=R_{0}>0
$$


where $R_{0}$ is a constant and

$$
\chi^{\mu \nu} \partial_{\mu} \partial_{\nu} \phi+\left(\eta^{\mu \nu}+\chi^{\mu \nu}\right) \Gamma_{\mu \nu}^{\lambda} \partial_{\lambda} \phi=0,
$$

which is a restriction on the deformation tensor $\chi^{\mu \nu}$. This condition allows to determine the mass by a conformal transformation. In fact, in the new frame, the equation is

$$
g^{\alpha \beta} \nabla_{\alpha} \partial_{\beta} \phi+\xi R \phi=0,
$$

which, in the case of a conformal transformation

$$
g_{\alpha \beta}=\Omega^{2} \eta_{\alpha \beta},
$$

becomes

$$
\Omega^{-2} \eta^{\alpha \beta}\left(\partial_{\alpha} \partial_{\beta} \phi+2 \Omega^{-1} \partial^{\gamma} \Omega \partial_{\gamma} \phi\right)-6 \xi \eta^{\alpha \beta} \Omega^{-3} \partial_{\alpha} \partial_{\beta} \Omega=0 .
$$

It is equivalent to a massive scalar field equation (in the first frame), if the condition

$$
2 \Omega^{-1} \partial^{\gamma} \Omega \partial_{\gamma} \phi-6 \xi \eta^{\alpha \beta} \Omega^{-1} \partial_{\alpha} \partial_{\beta} \Omega=m^{2},
$$

holds. Since Eq. (175) is linear in $\phi$, we can define $\phi=$ $e^{i \lambda_{\alpha} x^{\alpha}}$. Eq. (176) becomes

$$
2 \Omega^{-1} \partial^{\gamma} \Omega \lambda_{\gamma}-6 \xi \eta^{\alpha \beta} \Omega^{-1} \partial_{\alpha} \partial_{\beta} \Omega=m^{2},
$$

then a solution is $\Omega=e^{i k_{\alpha} x^{\alpha}}$ and the mass depends on the combination

$$
m^{2}=6 \xi \eta^{\alpha \beta} k_{\alpha} k_{\beta}-2 \eta^{\alpha \beta} k_{\alpha} \lambda_{\beta} .
$$

In this example, the deformation is a specific complex function, but we can extend this result to any function by taking

$$
\Omega(x)=\int \tilde{\Omega}(k) e^{i k x} d^{4} k,
$$

and also non-constant masses can be is obtained being

$$
m^{2}=\frac{\int \tilde{\Omega}(k)\left(6 \xi \eta^{\alpha \beta} k_{\alpha} k_{\beta}-2 \eta^{\alpha \beta} k_{\alpha} \lambda_{\beta}\right) e^{i k x} d^{4} k}{\int \tilde{\Omega}(k) e^{i k x} d^{4} k} .
$$

These results mean that a geometrical definition of mass is always possible. It can be induced by the conformal transformation $\Omega$ which is a restriction of the deformations group $G L(4)$. Summarizing we have shown that:

- the fifth dimension of a reduction mechanism from $5 \mathrm{D}$ to $4 \mathrm{D}$-manifolds can be interpreted as a mass generator:

- the further degrees of freedom coming out from Extended Theories of Gravity (i.e. extensions of GR) have a physical meaning and cannot be simply gauged away;

- as soon as particles acquire masses, $G L(4)$ can induce symmetry breakings giving rise to the observed interactions.

In the next section, we will discuss and classify the further degrees of freedom related to the Extended Gravity. The aim is to show how they could be related to a sort of Higgs-like mechanism.

\section{THE CLASSIFICATION OF GRAVITATIONAL MODES IN EXTENDED GRAVITY}

\section{A. Massive and massless modes of gravitational field}

The above considerations demonstrate that any reduction scheme from 5D to 4D induces effective theories of gravity where further gravitational modes have to be taken into account. In this view, GR is an exception including only massless tensor modes. Furthermore, GR is a degenerate theory where Hessian determinant is null preventing any quantization approach. With this situation is mind, it is natural to take into account further gravitational modes, emerging from Extended Gravity, that are usually discarded. Such modes could have interesting effects at ultra-violet and infra-red scales and could play an important role both in the Standard Model of particles and in gravitational radiation [72]. In particular, they could be connected to the symmetry breaking in the high energy limit (and investigated at LHC) and have a signature in the cosmological stochastic background of gravitational waves 109]. We want to show that such statements are very general and only GR shows two polarization modes (being a degenerate, constrained theory). As we are going to demonstrate, a generic Extended Theory of Gravity, constructed with a function of curvature invariants, shows six gravitational polarizations consistently with the Riemann theorem [56].

Assuming any curvature invariant other than the Ricci scalar 3 a generic $4 \mathrm{D}$-effective action for the gravitational interaction is

$$
\mathcal{A}=\int d^{4} x \sqrt{-g} f(R, P, Q)
$$

where

$$
\begin{aligned}
P & \equiv R_{\alpha \beta} R^{\alpha \beta} \\
Q & \equiv R_{\alpha \beta \gamma \delta} R^{\alpha \beta \gamma \delta}
\end{aligned}
$$

Varying with respect to the metric one gets the field equations:

$$
\begin{aligned}
F G_{\mu \nu}= & \frac{1}{2} g_{\mu \nu}(f-R F)-\left(g_{\mu \nu} \square-\nabla_{\mu} \nabla_{\nu}\right) F \\
& -2\left(f_{P} R_{\mu}^{\alpha} R_{\alpha \nu}+f_{Q} R_{\alpha \beta \gamma \mu} R_{\nu}^{\alpha \beta}\right) \\
& -g_{\mu \nu} \nabla_{\alpha} \nabla_{\beta}\left(f_{P} R^{\alpha \beta}\right)-\square\left(f_{P} R_{\mu \nu}\right) \\
& +2 \nabla_{\alpha} \nabla_{\beta}\left(f_{P} R_{(\mu}^{\alpha} \delta^{b} \text { eta }{ }_{\nu)}+2 f_{Q} R_{(\mu \nu)}^{\alpha}\right)
\end{aligned}
$$

3 We restrict to fourth-order theories which give the main contributions in a renormalization process, but we can extended the following considerations to any higher-order theory involving generic powers of the $\square$-operator. 
where we have set

$$
F \equiv \frac{\partial f}{\partial R}, \quad f_{P} \equiv \frac{\partial f}{\partial P}, \quad f_{Q} \equiv \frac{\partial f}{\partial Q}
$$

and, as above, $\square=g^{\alpha \beta} \nabla_{\alpha} \nabla_{\beta}$ is the d'Alembert operator. The notation $T_{(\mu \nu)}=\frac{1}{2}\left(T_{\mu \nu}+T_{\nu \mu}\right)$ denotes symmetrization with respect to the indices $(\mu, \nu)$.

Taking the trace of Eq. (183), we find:

$$
\begin{aligned}
& \square\left(F+\frac{f_{P}}{3} R\right)= \\
& \frac{1}{3}\left[2 f-R F-2 \nabla_{a} \nabla_{b}\left(\left(f_{P}+2 f_{Q}\right) R^{\alpha \beta}\right)+\right. \\
& \left.-2\left(f_{P} P+f_{Q} Q\right)\right]
\end{aligned}
$$

Expanding the third term on the r.h.s. of (185) and using the Bianchi identity $G_{; \beta}^{\alpha \beta}=0$, we get:

$$
\begin{aligned}
& \square\left(F+\frac{2}{3}\left(f_{P}+f_{Q}\right) R\right)=\frac{1}{3} \times \\
& {\left[2 f-R F-2 R^{\alpha \beta} \nabla_{\alpha} \nabla_{\beta}\left(f_{P}+2 f_{Q}\right)-R \square\left(f_{P}+2 f_{Q}\right)\right.} \\
& \left.-2\left(f_{P} P+f_{Q} Q\right)\right]
\end{aligned}
$$

If we define

$$
\begin{aligned}
\phi & \equiv F+\frac{2}{3}\left(f_{P}+f_{Q}\right) R \\
\frac{d V}{d \phi} & \equiv \text { r.h.s. of } 1186
\end{aligned}
$$

then we get a Klein-Gordon equation for the scalar field $\phi$ :

$$
\square \phi=\frac{d V}{d \phi} .
$$

Considering the discussion in previous section, it is clear that the scalar field $\phi$, defined in $4 \mathrm{D}$, assumes the role of a field induced by the further degrees of freedom of Extended Gravity. Obviously, $\phi$ is identically zero in GR.

In order to classify the gravitational modes that can be obtained from this approach, we need to linearize around the Minkowski background. As discussed above, this means to take into account deformations. According to the above results, we assume

$$
\begin{aligned}
g_{\mu \nu} & =\eta_{\mu \nu}+h_{\mu \nu} \\
\phi & =\phi_{0}+\delta \phi
\end{aligned}
$$

Then, from Eq. (187), we get

$$
\delta \phi=\delta F+\frac{2}{3}\left(\delta f_{P}+\delta f_{Q}\right) R_{0}+\frac{2}{3}\left(f_{P 0}+f_{Q 0}\right) \delta R
$$

where $R_{0} \equiv R\left(\eta_{\mu \nu}\right)=0$ and similarly $f_{P 0}=\left.\frac{\partial f}{\partial P}\right|_{\eta_{\mu \nu}}$ which is either constant or zero. Note that the index 0 indicates evaluation with respect to the Minkowski metric, that means no deformation. $\delta R$ denotes the first order perturbation on the Ricci scalar which, along with the perturbed parts of the Riemann and Ricci tensors, are given by:

$$
\begin{aligned}
\delta R_{\mu \nu \rho \sigma} & =\frac{1}{2}\left(\partial_{\rho} \partial_{\nu} h_{\mu \sigma}+\partial_{\sigma} \partial_{\mu} h_{\nu \rho}-\partial_{\sigma} \partial_{\nu} h_{\mu \rho}-\partial_{\rho} \partial_{\mu} h_{\nu \sigma}\right) \\
\delta R_{\mu \nu} & =\frac{1}{2}\left(\partial_{\sigma} \partial_{\nu} h_{\mu}^{\sigma}+\partial_{\sigma} \partial_{\mu} h_{\nu}^{\sigma}-\partial_{\mu} \partial_{\nu} h-\square h_{\mu \nu}\right) \\
\delta R & =\partial_{\mu} \partial_{\nu} h^{\mu \nu}-\square h
\end{aligned}
$$

where $h=\eta^{\mu \nu} h_{\mu \nu}$. The first term of Eq. (190) is

$$
\delta F=\left.\frac{\partial F}{\partial R}\right|_{0} \delta R+\left.\frac{\partial F}{\partial P}\right|_{0} \delta P+\left.\frac{\partial F}{\partial Q}\right|_{0} \delta Q
$$

However, since $\delta P$ and $\delta Q$ are second order, we get $\delta F \simeq$ $F_{, R 0} \delta R$ and

$$
\delta \Phi=\left(F_{, R 0}+\frac{2}{3}\left(f_{P 0}+f_{Q 0}\right)\right) \delta R
$$

Finally, from Eq. (186) we get the Klein-Gordon equation for the scalar perturbation $\delta \phi$

$$
\begin{aligned}
\square \delta \phi= & \frac{1}{3} \frac{F_{0}}{F_{, R 0}+\frac{2}{3}\left(f_{P 0}+f_{Q 0}\right)} \delta \phi- \\
& \frac{2}{3} \delta R^{\alpha \beta} \partial_{\alpha} \partial_{\beta}\left(f_{P 0}+2 f_{Q 0}\right)-\frac{1}{3} \delta R \square\left(f_{P 0}+2 f_{Q 0}\right) \\
= & m_{s}^{2} \delta \phi
\end{aligned}
$$

The last two terms in the first line are actually zero since the terms $f_{P 0}, f_{Q 0}$ are constants and we have defined the scalar mass as $m_{s}^{2} \equiv \frac{1}{3} \frac{F_{0}}{F_{, R 0}+\frac{2}{3}\left(f_{P 0}+f_{Q 0}\right)}$.

Perturbing the field equations (183) we get:

$$
\begin{aligned}
& F_{0}\left(\delta R_{\mu \nu}-\frac{1}{2} \eta_{\mu \nu} \delta R\right)= \\
& -\left(\eta_{\mu \nu} \square-\partial_{\mu} \partial_{\nu}\right)\left(\delta \phi-\frac{2}{3}\left(f_{P 0}+f_{Q 0}\right) \delta R\right) \\
& -\eta_{\mu \nu} \partial_{\alpha} \partial_{\beta}\left(f_{P 0} \delta R^{\alpha \beta}\right)-\square\left(f_{P 0} \delta R_{\mu \nu}\right) \\
& +2 \partial_{\alpha} \partial_{\beta}\left(f_{P 0} \delta R_{(\mu}^{\alpha} \delta_{\nu)}^{\beta}+2 f_{Q 0} \delta R_{(\mu \nu)}^{\alpha}\right)
\end{aligned}
$$

It is convenient to work in Fourier space where the following substitutions have to be operated: $\partial_{\gamma} h_{\mu \nu} \rightarrow i k_{\gamma} h_{\mu \nu}$ and $\square h_{\mu \nu} \rightarrow-k^{2} h_{\mu \nu}$. The above equation becomes

$$
\begin{aligned}
& F_{0}\left(\delta R_{\mu \nu}-\frac{1}{2} \eta_{\mu \nu} \delta R\right)= \\
& \left(\eta_{\mu \nu} k^{2}-k_{\mu} k_{\nu}\right)\left(\delta \phi-\frac{2}{3}\left(f_{P 0}+f_{Q 0}\right) \delta R\right) \\
& +\eta_{\mu \nu} k_{\alpha} k_{\beta}\left(f_{P 0} \delta R^{\alpha \beta}\right)+k^{2}\left(f_{P 0} \delta R_{\mu \nu}\right) \\
& -2 k_{a} k_{b}\left(f_{P 0} \delta R_{(\mu}^{a} \delta_{\nu)}^{b}\right)-4 k_{\alpha} k_{\beta}\left(f_{Q 0} \delta R_{(\mu \nu)}^{\alpha}{ }^{\beta}\right)
\end{aligned}
$$


We can rewrite the metric perturbation as

$$
h_{\mu \nu}=\bar{h}_{\mu \nu}-\frac{\bar{h}}{2} \eta_{\mu \nu}+\eta_{\mu \nu} h_{f}
$$

and impose the standard gauge conditions $\partial_{\mu} \bar{h}^{\mu \nu}=0$ and $\bar{h}=0$. The first of these conditions implies that $k_{\mu} \bar{h}^{\mu \nu}=0$ while the second gives

$$
\begin{aligned}
h_{\mu \nu} & =\bar{h}_{\mu \nu}+\eta_{\mu \nu} h_{f} \\
h & =4 h_{f}
\end{aligned}
$$

Inserting into the perturbed curvature quantities, we get

$$
\begin{aligned}
\delta R_{\mu \nu} & =\frac{1}{2}\left(2 k_{\mu} k_{\nu} h_{f}+k^{2} \eta_{\mu \nu} h_{f}+k^{2} \bar{h}_{\mu \nu}\right) \\
\delta R & =3 k^{2} h_{f} \\
k_{\alpha} k_{\beta} \delta R_{(\mu \nu)}^{\alpha{ }^{\beta}} & =-\frac{1}{2}\left(\left(k^{4} \eta_{\mu \nu}-k^{2} k_{\mu} k_{\nu}\right) h_{f}+k^{4} \bar{h}_{\mu \nu}\right) \\
k_{\alpha} k_{\beta} \delta R_{(\mu}^{\alpha} \delta_{\nu)}^{\beta} & =\frac{3}{2} k^{2} k_{\mu} k_{\nu} h_{f}
\end{aligned}
$$

Substituting Eqs. (196)-198) into (195) and after some algebra we get:

$$
\begin{aligned}
& \frac{1}{2}\left(k^{2}-k^{4} \frac{f_{P 0}+4 f_{Q 0}}{F_{0}}\right) \bar{h}_{\mu \nu}= \\
& \left(\eta_{\mu \nu} k^{2}-k_{\mu} k_{\nu}\right) \frac{\delta \phi}{F_{0}}+\left(\eta_{\mu \nu} k^{2}-k_{\mu} k_{\nu}\right) h_{f}
\end{aligned}
$$

Defining $h_{f} \equiv-\frac{\delta \phi}{F_{0}}$ we find the equation for the perturbations:

$$
\left(k^{2}+\frac{k^{4}}{m_{\text {spin } 2}^{2}}\right) \bar{h}_{\mu \nu}=0
$$

where $m_{\text {spin } 2}^{2} \equiv-\frac{F_{0}}{f_{P 0}+4 f_{Q 0}}$. From Eq. (193) we get:

$$
\square h_{f}=m_{s}^{2} h_{f}
$$

From Eq. 200 it is easy to see that we have a modified dispersion relation which corresponds to a massless spin-2 field $\left(k^{2}=0\right)$ and a massive spin-2 field $k^{2}=\frac{F_{0}}{\frac{1}{2} f_{P 0}+2 f_{Q 0}} \equiv-m_{\operatorname{spin} 2}^{2}$. To see this better this point, let us note that the propagator for $\bar{h}_{\mu \nu}$ can be rewritten as

$$
G(k) \propto \frac{1}{k^{2}}-\frac{1}{k^{2}+m_{\text {spin } 2}^{2}}
$$

Clearly the second term has the opposite sign, which indicates the presence of a ghost energy mode (see also [67 69]).

As a "sanity check", we can see that for the GaussBonnet term $\mathcal{L}_{G B}=Q-4 P+R^{2}$ we have $f_{P 0}=-4$ and $f_{Q 0}=1$. Then, Eq. (200) simplifies to $k^{2} \bar{h}_{\mu \nu}=0$ and, in this case, we have no negative energy modes as expected.
The solutions of Eqs. (200) and (201) can be written in terms of plane waves

$$
\begin{gathered}
\bar{h}_{\mu \nu}=A_{\mu \nu}(\vec{p}) \cdot \exp \left(i k^{\alpha} x_{\alpha}\right)+c c \\
h_{f}=a(\vec{p}) \cdot \exp \left(i q^{\alpha} x_{\alpha}\right)+c c
\end{gathered}
$$

where

$$
\begin{aligned}
k^{\alpha} \equiv\left(\omega_{m_{\text {spin } 2}}, \vec{p}\right) & \omega_{m_{\text {spin } 2}}=\sqrt{m_{s p i n 2}^{2}+p^{2}} \\
q^{\alpha} \equiv\left(\omega_{m_{s}}, \vec{p}\right) & \omega_{m_{s}}=\sqrt{m_{s}^{2}+p^{2}}
\end{aligned}
$$

and where $m_{\operatorname{spin} 2}$ is zero (non-zero) in the case of massless (massive) spin-2 mode and the polarization tensors $A_{\mu \nu}(\vec{p})$ can be found in Ref. [70] (see equations (21)(23)). Eqs. (200) and (203), contain the equation and the solution for the standard gravitational waves of GR 71] plus massive spin 2 terms. Eqs. (201) and (204) are respectively the equation and the solution for the massive scalar mode (see also 72]).

The fact that the dispersion law for the modes of the massive field $h_{f}$ is not linear has to be emphasized. The velocity of every "ordinary" (arising from GR) mode $\bar{h}_{\mu \nu}$ is the light speed $c$, but the dispersion law (the second of Eq. (205) for the modes of $h_{f}$ is that of a massive field which can be seen as a wave-packet. Also, the groupvelocity of a wave-packet of $h_{f}$, centered in $\vec{p}$, is

$$
\overrightarrow{v_{G}}=\frac{\vec{p}}{\omega}
$$

which is exactly the velocity of a massive particle with mass $m$ and momentum $\vec{p}$. From the second of Eqs. (205) and Eq. (206) it is straightforward to obtain:

$$
v_{G}=\frac{\sqrt{\omega^{2}-m^{2}}}{\omega} .
$$

This means that the speed of the wave-packet is

$$
m=\sqrt{\left(1-v_{G}^{2}\right)} \omega
$$

Summarizing these results, we can say that considering Extended Theories of Gravity (which we have generically assumed as analytic functions of curvature invariants) more gravitational modes than the standard massless ones of GR have to be taken into account. These further modes can be derived from metric deformations and characterized as propagating particles.

\section{B. Polarization states}

As we have seen, there are solutions of Eq. (200) depending on the value of $k^{2}$. We have $k^{2}=0$ modes 
that corresponds to a massless spin-2 field with two independent polarizations (the standard polarization states of GR). If $k^{2} \neq 0$, we have massive spin- 2 ghost modes and there are five independent polarization tensors. The number of polarizations can be easily achieved by the formula of spin degeneration $d=(2 s+1)$ [70]. A further scalar mode comes out from Eq.(201). Due to the above formula for spin degeneration, it gives one polarization state.

Let us first consider the case where the spin-2 field is massless. Assuming $\vec{p}$ in the $z$ direction, a gauge in which only $A_{11}, A_{22}$, and $A_{12}=A_{21}$ are different from zero can be chosen. The condition $\bar{h}=0$ gives $A_{11}=-A_{22}$. In this frame, we may take the bases of polarizations defined a:4

$$
\begin{gathered}
e_{\mu \nu}^{(+)}=\frac{1}{\sqrt{2}}\left(\begin{array}{ccc}
1 & 0 & 0 \\
0 & -1 & 0 \\
0 & 0 & 0
\end{array}\right), \quad e_{\mu \nu}^{(\times)}=\frac{1}{\sqrt{2}}\left(\begin{array}{lll}
0 & 1 & 0 \\
1 & 0 & 0 \\
0 & 0 & 0
\end{array}\right) \\
e_{\mu \nu}^{(s)}=\frac{1}{\sqrt{2}}\left(\begin{array}{lll}
0 & 0 & 0 \\
0 & 0 & 0 \\
0 & 0 & 1
\end{array}\right)
\end{gathered}
$$

Inserting these equations in Eq. (196), it results

$$
\begin{aligned}
h_{\mu \nu}(t, z) & =A^{+}(t-z) e_{\mu \nu}^{(+)}+A^{\times}(t-z) e_{\mu \nu}^{(\times)} \\
& +h_{s}\left(t-v_{G} z\right) e_{\mu \nu}^{s}
\end{aligned}
$$

The terms $A^{+}(t-z) e_{\mu \nu}^{(+)}+A^{\times}(t-z) e_{\mu \nu}^{(\times)}$describe the two standard polarizations of gravitational waves which arise from GR, while the term $h_{s}\left(t-v_{G} z\right) \eta_{\mu \nu}$ is the massive field arising from a generic Extended Theory of Gravity where further degrees of freedom can be represented by a scalar field.

When the spin-2 field is massive, we have six polarization (five due to the spin 2 and one due to the spin 0 ). Possible bases of polarizations are

$$
\begin{array}{cc}
e_{\mu \nu}^{(+)}=\frac{1}{\sqrt{2}}\left(\begin{array}{ccc}
1 & 0 & 0 \\
0 & -1 & 0 \\
0 & 0 & 0
\end{array}\right), & e_{\mu \nu}^{(\times)}=\frac{1}{\sqrt{2}}\left(\begin{array}{lll}
0 & 1 & 0 \\
1 & 0 & 0 \\
0 & 0 & 0
\end{array}\right) \\
e_{\mu \nu}^{(B)}=\frac{1}{\sqrt{2}}\left(\begin{array}{ccc}
0 & 0 & 1 \\
0 & 0 & 0 \\
1 & 0 & 0
\end{array}\right), & e_{\mu \nu}^{(C)}=\frac{1}{\sqrt{2}}\left(\begin{array}{lll}
0 & 0 & 0 \\
0 & 0 & 1 \\
0 & 1 & 0
\end{array}\right) \\
e_{\mu \nu}^{(D)}=\frac{\sqrt{2}}{3}\left(\begin{array}{ccc}
\frac{1}{2} & 0 & 0 \\
0 & \frac{1}{2} & 0 \\
0 & 0 & -1
\end{array}\right), & e_{\mu \nu}^{(s)}=\frac{1}{\sqrt{2}}\left(\begin{array}{lll}
0 & 0 & 0 \\
0 & 0 & 0 \\
0 & 0 & 1
\end{array}\right) .
\end{array}
$$

\footnotetext{
4 The polarizations are defined in the 3 -space, not in a space-time. Each polarization mode is orthogonal to one another and is normalized $e_{\mu \nu} e^{\mu \nu}=2 \delta$. Note that other modes are not traceless, in contrast to the ordinary plus and cross polarization modes in GR.
}
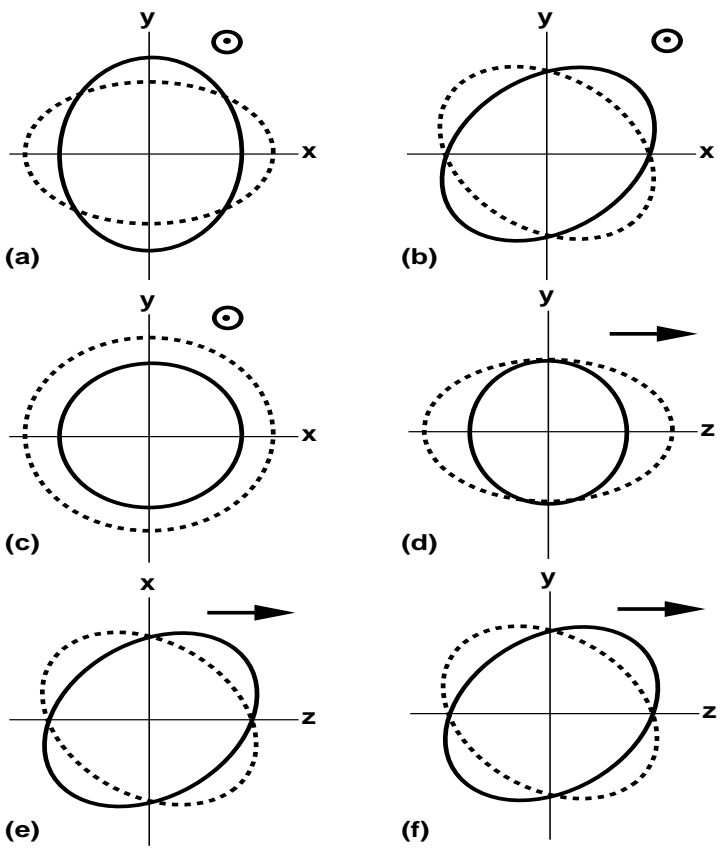

Figure 1. The possible polarization modes of gravitons. The picture shows the displacement that each mode induces on a sphere of test particles. The mode propagates out of the plane in (a), (b), (c), and it propagates in the plane in (d), (e) and (f). In (a) and (b) we have respectively the plus mode and cross mode, in (c) the scalar mode, in (d), (e) and (f) the extra D, B and C modes induced by massive spin- 2 gravitons. This behavior could be, in principle, observed also for a beam of particles undergoing this interaction at suitable scales.

The total amplitude can be written in terms of the 6 polarization states as

$$
\begin{aligned}
& h_{\mu \nu}(t, z)=A^{+}\left(t-v_{G_{s 2}} z\right) e_{\mu \nu}^{(+)}+A^{\times}\left(t-v_{G_{s 2}} z\right) e_{\mu \nu}^{(\times)} \\
& +B^{B}\left(t-v_{G_{s 2}} z\right) e_{\mu \nu}^{(B)}+C^{C}\left(t-v_{G_{s 2}} z\right) e_{\mu \nu}^{(C)} \\
& +D^{D}\left(t-v_{G_{s 2}} z\right) e_{\mu \nu}^{(D)}+h_{s}\left(t-v_{G} z\right) e_{\mu \nu}^{s} .
\end{aligned}
$$

where $v_{G_{s 2}}$ is the group velocity of the massive spin-2 field and is given, as above, by

$$
v_{G_{s 2}}=\frac{\sqrt{\omega^{2}-m_{s 2}^{2}}}{\omega}
$$

The first two polarizations are the same as in the massless case, inducing tidal deformations on the $\mathrm{x}-\mathrm{y}$ plane. In Fig.1, we illustrate how each polarization could affect test masses arranged along a circle.

\section{The interpretation of gravitational ghost modes}

The presence of negative energy modes could seem a pathology from a purely quantum-mechanical point of 
view. However, there are several interpretations that have to be taken into account for such phenomena. A ghost mode can be viewed as either a particle state of positive energy and negative probability density, or a positive probability density state with a negative energy. In the first case, allowing the presence of such a particle will quickly induce violation of unitarity. The negative energy scenario leads to a theory where there is no energy minimum and the system thus becomes unstable with growing amplitudes. The vacuum can decay into pairs of ordinary and ghost gravitons leading to an instability. In other words, such a dynamics induces a symmetry breaking and this fact can be directly related to the fact that $G L(4)$ is a non-unitary group. In order to regularize the theory, dynamical ghost modes can be cancelled out taking into account yet higher derivative terms. This leads to a higher order effective action as the one in Eq. (107) which, in principle, is an infinite order effective action 5 .

One way out of such problems is to ask for a very weak coupling of the ghost with the other particles in the theory. In this case, the decay rate of the vacuum becomes comparable to the inverse of a suitable length scale. If such a scale is extremely large, the present vacuum state will appear to be sufficiently stable. This option is viable when ghost states, due to a different interaction length, couple differently than the ordinary massless gravitons with the other forms of matter.

Another interpretation is to assume that this picture does not hold up to arbitrarily high energies but, at some cutoff scale $M_{\text {cutoff }}$, the theory modifies appropriately to ensure a ghost-free behavior and a stable ground state. This could happen, for example, if the Lorentz invariance is violated at a certain $M_{\text {cutoff }}$, thereby restricting any potentially harmful decay rates. This possibility could be extremely interesting for the investigations at LHC, being a sort of gravitationally induced Higgs-like mechanism [73, 74]. .

However, we have to point out that Extended Theories of Gravity could not hold up to arbitrary high energies. Such models are plagued, at fundamental quantum level, by the same problems as ordinary GR, but Extended Theories of Gravity are renormalizable at one loop-level [104] . They are not proper candidates for a full quantum gravity theory (in canonical sense) but the corresponding ghost particle interpretation (virtual massive modes) is a useful approach to address, at lower energies than Planck scales, shortcomings of Standard Model.

At semi-classical level, the perturbation $h_{\mu \nu}$ (deformation) is a tensor representing the "stretching" of spacetime away from flatness. A ghost mode then makes sense as just another way of propagating this perturbation of the space-time geometry, one which carries the opposite

\footnotetext{
${ }^{5}$ In this case, we should consider higher order powers in the $\square$ operator.

6 The largest length is the Hubble scale. This gives rise to the weakest physical coupling.
}

sign in the propagator than an ordinary massive graviton would.

Viewed in this way, the presence of the massive ghost graviton will induce the same effects as an ordinary massive graviton transmitting the perturbation, but with the opposite sign in the displacement. Tidal stretching from a polarized wave on the polarization plane will be turned into shrinking and vice-versa. This signal will, at the end, be a superposition of the displacements coming from the ordinary massless spin-2 graviton and the massive ghost. Since these modes induce two competing effects, this fact would lead to a less pronounced signal than the one we would expect if the ghost mode was absent, setting in this way less severe constraints on the theory. On the other hand, treating ghost modes as small perturbations could be not sensible. As stated above, the dynamical ghost modes could be cancelled by other higher derivative terms. In that case, nonetheless, it might still make sense to analyze the impact on propagation owing to virtual massive mode effects (Yukawa terms) on the massless modes. However, the presence of the new modes will also affect the total energy density carried by the graviton and could induce new effects at ultraviolet scales, as we will discuss below. To conclude, effects and symmetry breaking discussed here could give rise to relevant signatures in colliding beams of particles (e.g. hadrons) at $\mathrm{TeV}$ scales.

\section{MASSIVE GRAVITATIONAL STATES AND THE INDUCED SYMMETRY BREAKING}

\section{A. A gravitational cut-off at TeV scales}

The above results could be interesting to investigate quantum gravity effects and symmetry breaking in the range between $\mathrm{GeV}$ and $\mathrm{TeV}$ scales. Such scales are actually investigated by the experiments at LHC. It is important to stress that any ultra-violet model of gravity (e.g. at $\mathrm{TeV}$ scales) have to explain also the observed weakness of gravitational effects at largest (infra-red) scales. This means that massless (or quasi-massless) modes have to be considered in any case.

The above 5D-action (79) is an example of higher dimensional action where the effective gravitational energy scale (Planck scale) can be "rescaled" according to Eqs. (98) and (99). In terms of mass, being $M_{p}^{2}=\frac{c \hbar}{G_{N}}$ the constraint coming from the ultra-violet limit of the theory $\left(10^{19} \mathrm{GeV}\right)$, we can set $M_{p}^{2}=M_{\sharp}^{D-2} V_{D-4}$, where $V_{D-4}$ is the "volume" coming from the extra dimension. It is easy to see that $V_{D-4}$, in the $5 \mathrm{D}$ case, is related to the fifth component of $\Phi$, that is $\psi\left(x_{4}\right)$ in Eq. 135. $M_{\sharp}$ is the above discussed cut-off mass that becomes relevant as soon as the Lorentz invariance is violated. Such a scale, in the context discussed here could be of the order $\mathrm{TeV}$.

As we have shown in Sec. IV it is quite natural to obtain effective theories containing scalar fields of gravitational origin. In this sense, $M_{\sharp}$ is the result of dimensional reduction. To be more explicit, the $4 \mathrm{D}$ dynamics is 
led by the effective potential $V(\phi)$ and the non-minimal coupling $F(\phi)$. Such functions could be experimentally tested since related to massive states. In particular, the effective Extended Gravity, produced by the reduction mechanism from $5 \mathrm{D}$ to $4 \mathrm{D}$, can be chosen as

$$
\mathcal{A}=\int d^{4} x \sqrt{-g}\left[-\frac{\phi^{2}}{2} R+\frac{1}{2} g^{\mu \nu} \partial_{\mu} \phi \partial_{\nu} \phi-V\right](213)
$$

plus contributions of ordinary matter terms. The potential for $\phi$ can be assumed as

$$
V(\phi)=\frac{M_{\sharp}^{2}}{2} \phi^{2}+\frac{\lambda}{4} \phi^{4},
$$

where a massive term and the self-interaction term are present. This is the standard choice of quantum field theory which perfectly fits with the arguments of dimensional reduction. Let us recall again that the scalar field $\phi$ is not put by hand into dynamics but it is given by the extra degrees of freedom of gravitational field generated by the reduction process in $4 \mathrm{D}$. It is easy to derive the vacuum expectation value of $\phi$, being

$$
M_{\sharp}^{2}=2 \lambda M_{p}^{2},
$$

which is a fundamental scale of the theory.

Some considerations are in order at this point. Such a scale has to be confronted with Higgs vacuum expectation value which is $246 \mathrm{GeV}$ and then with the hierarchy problem. If $M_{\sharp}$ is larger than Higgs mass, the problem is obviously circumvented. It is important to recall that hierarchy problem occurs when couplings and masses of effective theories are very different than the parameters measured by experiments. This happen since measured parameters are related to the fundamental parameters by renormalization and fine cancellations between the fundamental quantities and the quantum corrections are necessary. The hierarchy problem is essentially a fine-tuning problem.

In particle physics, the question is why the weak force is stronger and stronger than gravity. Both of these forces involve constants of nature, Fermi's constant for the weak force and Newton's constant for gravity. From the Standard Model, it appears that Fermi's constant is unnaturally large and should be closer to Newton's constant, unless there is a fine cancellation between the bare value of Fermi's constant and the quantum corrections to it.

More technically, the question is why the Higgs boson is so much lighter than the Planck mass (or the grand unification energy). In fact, researchers are searching for Higgs masses ranging from 115 up to $350 \mathrm{GeV}$ with different selected decay channels from $b \bar{b}$ to $t \bar{t}$ (see for example [112] and refereces therein). One would expect that the large quantum contributions to the square of the Higgs boson mass would inevitably make the mass huge, comparable to the scale at which new physics appears, unless there is an incredible fine-tuning cancellation between the quadratic radiative corrections and the bare mass. With this state of art, the problem cannot be formulated in the context of the Standard Model where the Higgs mass cannot be calculated. In a sense, the problem is solvable if, in a given effective theory of particles, where the Higgs boson mass is calculable, there are no fine-tunings. If one accepts the big-desert assumption and the existence of a hierarchy problem, some new mechanism (at Higgs scale) becomes necessary to avoid fine-tunings.

The model which we are discussing contains a "running" scale that could avoid to set precisely the Higgs scale. If the mass of the field $\phi$ is in $\mathrm{TeV}$ region, there is no hierarchy problem being $\phi$ a gravitational scale. In this case, the Standard Model holds up plus an extended gravitational sector derived from the fifth dimension.

In other words, the Planck scale can be dynamically derived from the vacuum expectation value of $\phi$. In some sense, our model, in its low energy realization, works like the model proposed by Antoniadis et al [76]. The Planck scale can be recovered, as soon as the coupling $\lambda$ is of the order $10^{-31}$. Action (213) (and the other Extended Gravity Theories discussed above) as effective models valid up to a cutoff scale of a few $M_{\sharp} \sim \mathrm{TeV}$ (see also [110, 115, 116]). The tiny value of $\lambda$, coming from the extra dimension, is in agreement with the above considerations allowing the presence of physical (quasi-) massless gravitons with very large interaction lengths.

As in [76], gravity is weak although the gravitational scale is low. Also the string theory limit corresponds to a large scalar field vacuum expectation value at $\mathrm{TeV}$. It is important to stress that, by a conformal transformation from the Jordan frame to the Einstein frame, the Planck scale is decoupled from the vacuum expectation of the scalar field $\phi$. However the scalar field redefinition has to preserve the vacuum of the theory. Besides, the gauge couplings and masses depend on the vacuum expectation value of $\phi$ and are dynamically determined. This means that Standard Model and Einstein Gravity (in the above conformal-affine sense) could be recovered without the hierarchy problem. As discussed in [111], it is possible to show that the operators generated by the self-interaction of the scalar field are of the form

$$
\frac{1}{M_{\sharp}^{N-4}} \lambda^{\frac{N}{2}} \phi^{N}
$$

and they are always suppressed by the small parameter $\lambda$ and do not destabilize the potential of the theory. This result holds also for perturbative corrections coming from quantum gravity.

Considering again the problem of mass generation, one can assume that particles of Standard Model have sizes related to the cut off, that is $M_{\sharp}^{-1}$, and their collisions could lead to the formation of bound states as in [76, 114]. Potentially, such a phenomenon could mimic the decay of semi-classical quantum black holes and, at lower energies, it could be useful to investigate substructures of Standard Model. This means that we should expect some strong scattering effects in the $\mathrm{TeV}$ region involving the coupling of $\phi$ to the Standard Model fields. The "signature" of this phenomenon could lead to polarization 
effects of the particle beam as discussed in the previous section. Furthermore the strong dynamics derived from the phenomenon could resemble compositeness as discussed in [79]. Furthermore, bounds on the production of mini-black holes can be derived from astroparticle physics [80 84]. In [81] a bound on the cross-section is

$$
\sigma_{\nu N \rightarrow B H+X}<\frac{0.5}{\mathrm{TeV}^{2}} \text {. }
$$

Assuming, in our case, the cross-section $\sigma=M_{\sharp}^{-2}$, we get a bound of $\mathrm{TeV}$-order. If the fundamental scale of our theory is of this order, strong scattering processes at LHC would have the cross-section

$$
\sigma_{(p p \rightarrow \text { grav.ghosts }+X)} \sim 1 \times 10^{7} \mathrm{fb}
$$

and would dominate the cross-sections expected from the Standard Model. In this case, the Higgs boson could not be detected and no hierarchy problem would be present.

\section{B. Gravitational and electroweak interactions}

The Higgs mechanism is an approach that allows: $i$ ) to generate the masses of electroweak gauge bosons; $i$ ) to preserve the perturbative unitarity of the S-matrix; iii) to preserve the renormalizability of the theory. The masses of the electroweak bosons can be written in a gauge invariant form using either the non-linear sigma model [22] or a gauge invariant formulation of the electroweak bosons. However if there is no propagating Higgs boson, quantum field amplitudes describing modes of the electroweak bosons grow too fast violating the unitarity around $\mathrm{TeV}$ scales 85 88. There are several ways in which unitarity could be restored but the Standard Model without a Higgs boson is non-renormalizable at perturbative level.

A possibility is that the weak interactions become strongly coupled at $\mathrm{TeV}$ scales and then the related gauge theory becomes unitary at non-perturbative level. Another possibility for models without a Higgs boson consists in introducing weakly coupled new particles to delay the unitarity problem into the multi $\mathrm{TeV}$ regime where the UV limit of the Standard Model is expected to become relevant. Dvali et al. [89] proposed that, as black holes in gravitational scattering, classical objects could form in the scattering of longitudinal W-bosons leading to unitary scattering amplitude.

These ideas are very intriguing and show several features of electroweak interactions. First of all, the Higgs mechanism is strictly necessary to generate masses for the electroweak bosons. Beside, some mechanisms can be unitary but not renormalizable or vice-versa. In summary, the paradigm is that three different criteria should be fulfilled: $i$ ) a gauge invariant generation of masses of electroweak bosons, $i i$ ) perturbative unitarity; $i i i)$ renormalizability of the theory.

Here we have proposed an alternative approach, based on Extended Theories of Gravity deduced from a 5Dmanifold where the Standard Model is fully recovered enlarging the gravitational sector but avoiding the Higgs boson and the hierarchy problem.

It is important to point out that, in both the nonlinear sigma model and in gauge invariant formulation of Standard Model, it is possible to define an action in terms of an expansion in the scale of the electroweak interactions $v$. The action can be written as

$$
\mathcal{A}=\mathcal{A}_{\text {SMw/oHiggs }}+\int d^{4} x \sum_{i} \frac{C_{i}}{v^{N}} O_{i}^{4+N},
$$

where $O_{i}^{4+N}$ are operators compatible with the symmetries of the model. The electroweak bosons are gauge invariant fields defined by

$$
\underline{W}_{\mu}^{i}=\frac{i}{2 g} \operatorname{Tr} \Omega^{\dagger} \stackrel{\leftrightarrow}{D}_{\mu} \Omega \tau^{i},
$$

with $D_{\mu}=\partial_{\mu}-i g B_{\mu}(x)$ and

$$
\Omega=\frac{1}{\sqrt{\phi^{\dagger} \phi}}\left(\begin{array}{cc}
\phi_{2}^{*} & \phi_{1} \\
-\phi_{1}^{*} & \phi_{2}
\end{array}\right)
$$

where

$$
\phi=\left(\begin{array}{c}
\phi_{1} \\
\phi_{2}
\end{array}\right)
$$

is a $S U(2)_{L}$ doublet scalar field which is considered to be a dressing field and does not need to propagate. The same approach can be applied to fermions [90, 92, 93].

The analogy between the effective action for the electroweak interactions (219) and that of Extended Gravity is striking. Considering only the leading terms, the above theory can be written as a Taylor series of the form

$$
f(R) \simeq \Lambda+f_{0}^{\prime} R+\frac{1}{2 !} f_{0}^{\prime \prime} R^{2}+\frac{1}{3 !} f_{0}^{\prime \prime \prime} R^{3}+\ldots .
$$

where the coefficients are the derivatives of $f(R)$ calculated at a certain value of $R$. Clearly, as shown in previous sections, the extra gravitational degrees of freedom can be suitably transformed in a scalar field $\phi$ which allows to avoid the hierarchy problem. Both electroweak theory and Extended Gravity have a dimensional energy scale which defines the strength of the interactions. The Planck mass sets the strength of gravitational interactions while the weak scale $\lambda$ determines the range and the strength of the electroweak interactions. As shown in the previous subsection, these scales can be compared at $\mathrm{TeV}$ energies.

In other words, the electroweak bosons are not gauge bosons in standard sense but they can be "derived" from the above further gravitational degrees of freedom. The local $S U(2)_{L}$ gauge symmetry is imposed at the level of the quantum fields. However there is a residual global $S U(2)$ symmetry, i.e. the custodial symmetry. In the case of gravitational theories formulated as the $G L(4)$ group of diffeomorphisms, tetrads are an unavoidable feature necessary to construct the theory. They are gauge 
fields which transform under the local Lorentz transformations $S O(3,1)$ and under general coordinate transformations, the metric $g_{\mu \nu}=e_{\mu}^{a} e_{\nu}^{b} \eta_{a b}$ which is the field that is being quantized, transforms under general coordinate transformations which is the equivalent of the global $S U(2)$ symmetry for the weak interactions (in our case the residual $G L(2) \supset S U(2))$. Such an analogy between the tetrad fields and the Higgs field is extremely relevant. As shown above for deformations, we can say that the Higgs field has the same role of the tetrads for the electroweak interactions while the electroweak bosons have the same role of the metric. Dynamics is given by deformations.

A gravitational action like (223) is, in principle, nonperturbatively renormalizable if, as shown by Weinberg, there is a non-trivial fixed point which makes the gravity asymptotically free [108]. This scenario implies that only a finite number of the Wilson coefficients in the effective action would need to be measured and the theory would thus be predictive and probed at LHC.

Measuring the strength of the electroweak interactions in the electroweak W-boson scattering could easily reveal a non-trivial running of the electroweak scale $v$. If an electroweak fixed point exists, an increase in the strength of the electroweak interactions could be found, as in the strongly interacting W-bosons scenario, before the electroweak interactions become very weak and eventually irrelevant in the fixed point regime. In analogy to the non-perturbative running of the non-perturbative Planck mass, it is possible to introduce an effective weak scale

$$
v_{e f f}^{2}=v^{2}\left(1+\frac{\omega}{8 \pi} \frac{\mu^{2}}{v^{2}}\right)
$$

where $\mu$ is an arbitrary mass scale, $\omega$ a non-perturbative parameter which determines the running of the effective weak scale and $v$ is the weak scale measured at low energies. If $\omega$ is positive, the electroweak interactions would become weaker with increasing center of mass energy. This asymptotically free weak interaction would be renormalizable at the non-perturbative level without having a propagating Higgs boson again in analogy to Extended Gravity.

The asymptotically free weak interaction scenario could also solve the unitarity problem of the standard model without a Higgs boson. In the standard model without a Higgs boson, there are five amplitudes contributing at tree-level to the scattering of two longitudinally polarized electroweak W-bosons. Summing these five amplitudes, one finds at order $s / M_{W}^{2}$

$$
\mathcal{A}\left(W_{L}^{+}+W_{L}^{-} \rightarrow W_{L}^{+}+W_{L}^{-}\right)=\frac{s}{v_{e f f}^{2}}\left(\frac{1}{2}+\frac{1}{2} \cos \theta\right)
$$

where $s$ is the center of mass energy squared and $\theta$ is the scattering angle. Clearly if $v_{\text {eff }}$ grows fast enough with energy, the ultra-violet behaviour of these amplitudes can be compensated and the summed amplitude can remain below the unitary bound. A similar proposal has been made to solve problems with unitarity in extradimensional models 94.

It is important to stress that our approach does not require new physics but to take only into account the whole budget of gravitational degrees of freedom. The monitoring of the strength of the electroweak interactions in the W-bosons scattering at LHC could establish the existence of a fixed-point in the weak interactions. Using the oneloop renormalization group of the weak scale could help in formalizing this picture [95]. To be more precise, let us consider the scale of electroweak interactions

$$
v(\mu)=v_{0}\left(\frac{\mu}{\mu_{0}}\right)^{\frac{\gamma}{16 \pi^{2}}}
$$

where

$$
\gamma=\frac{9}{4}\left(\frac{1}{5} g_{1}^{2}+g_{2}^{2}\right)-Y_{2}(S)
$$

and

$$
Y_{2}(S)=\operatorname{Tr}\left(3 \mathrm{Y}_{u}^{\dagger} \mathrm{Y}_{u}+3 \mathrm{Y}_{d}^{\dagger} \mathrm{Y}_{d}+\mathrm{Y}_{e}^{\dagger} \mathrm{Y}_{e}\right)
$$

where $\mathrm{Y}_{i}$ are the respective Yukawa matrices. If the theory is in the perturbative regime e.g. at $m_{W}$, the Yukawa coupling of the top dominates since at this scale $g_{1}=0.31$ and $g_{2}=0.65$ and $\gamma$ is negative. In this case, the scale of the weak interactions become smaller. If the weak interactions become strongly coupled at $\mathrm{TeV}$ region, $g_{2}$ becomes large and $\gamma$ is expected to become positive. We obtain the expected running and the weak scale becomes larger. This is not possible in the framework of a perturbative approach. This result could represent a "signature" for the approach presented here. However, we stress once again that there are indications of a nontrivial fixed point for the non-linear sigma model using exact renormalization group techniques [96, 97]. In conclusion, the unitarity problem of the weak interactions could be fixed by a non-trivial fixed point in the renormalization group of the weak scale. A similar mechanism could also fix the unitarity problem for fermions masses 98 102 if their masses are not generated by the standard Higgs mechanism but in the same way considered here (let us remind that also $S U(3)$ could be generated by the splitting of $G L(4)$-group). In the case of electroweak interactions this approach could be soon checked at LHC but good indications are also available for QCD [113].

\section{DISCUSSION AND CONCLUSIONS}

The goal of this work is to give a unification scheme of fundamental interactions based on a well defined nonperturbative dynamics, the non-introduction of ad hoc hypotheses and the consideration of the minimal necessary number of free parameters and dimensions.

As general preliminaries, we have discussed the conformal-affine structure of fiber bundles showing that, 
in principle, different Extended Theories of Gravity can be conformally related each other. After, we have discussed the group structure in $5 \mathrm{D}$ and in $4 \mathrm{D}$-spaces showing how the group of diffeomorphisms $G L(4)$ works from a reduction procedure from $5 \mathrm{D}$ to $4 \mathrm{D}$. Such a group can be suitably split generating the fundamental groups of physical interactions. In this respect, the group splitting is

$$
\underbrace{\underbrace{4 \times 4}}_{\text {diffeom. }} \underbrace{\underbrace{2}-1}_{\text {gluons }} \underbrace{S U(3)}_{\text {vec. bosons }} \otimes \underbrace{\underbrace{2}-1(2)}_{\text {photon }} \otimes \underbrace{2^{2}-1}_{\text {gravitons }}
$$

with further gravitational degrees of freedom.

After we have shown that the space-time deformations in 4D have a conformal structure and the $G L(4)$-algebra.

Starting with these mathematical tools, we proposed a unification scheme based on the assumption that a 5Dspace can be defined where conservation laws are always and absolutely conserved. Such a General Conservation Principle [42] holds since we ask for the validity of the 5D-Bianchi identities which must be always non-singular and invariant for every diffeomorphism. The $5 \mathrm{D}$-space is a smooth, connected and compact manifold where we can derive field equations, geodesic equations and a globally defined Lorentz structure. The standard physics emerges as soon as we reduce from $5 \mathrm{D}$ to $4 \mathrm{D}$-space recovering the $G L(4)$-group of diffeomorphisms. By a reduction procedure one is capable of generating the masses of particles and their organization in families. The byproduct is an effective theory of gravity in $4 \mathrm{D}$ where further gravitational degrees of freedom naturally emerge, induced by the fifth dimension. By this dynamics, we do not recover the Standard GR but Extended Theories of Gravity where non-minimal coupling, scalar field self-interaction potentials and higher-order curvature terms have to be considered. These theories can be confronted and related, as we have seen, by conformal transformations.

The main feature is that higher-order terms or induced scalar fields enlarge the gravitational sector giving rise to massless, massive spin-2 gravitons and massive spin- 0 gravitons. Such gravitational modes results in 6 polarizations, according to the prescription of the Riemann theorem stating that in a given $N$-dimensional space, $N(N-1) / 2$ degrees of freedom are possible. The massive spin-2 gravitational states are ghost particles. Their role result relevant as soon as we can define a cut-off mass at $\mathrm{TeV}$ scale (the vacuum state of the scalar field) that allows both to circumvent the hierarchy problem and the detection of the Higgs boson. In such a case, the Standard Model of particles should be confirmed without recurring to perturbative, renormalizable schemes involving new particles. The weakness of self-interaction coupling would guarantee the fact that gravity could be compared, at $\mathrm{TeV}$ scale, with electroweak interaction.

However, some crucial points have to be considered in order to improve of the proposed approach. The main goal of our scenario is that the Standard Model of particles could be generated by the effective gravitational interactions coming from higher dimensions. In particular the gauge symmetries and mass generations could be achieved starting from conservation laws in $5 \mathrm{D}$. It is important to stress that the Standard Model does not mean only the gauge interaction but also quarks and leptons with their mass matrices that have to be exactly addressed. In particular, the fermion sector has to be recovered.

It is well-known that the standard gauge interactions contains the chiral gauge interactions, which, in our picture, have to be generated from the gravitational interactions otherwise there is no possibility to distinguish between the left-handed and the right-handed particles. In particular, the $S U(2)$ part of the standard gauge interactions, generated from $G L(4)$, has to be chiral and, consequently, fermions acquire a chiral representation. To this end, torsion fields have to be incorporated for the following reasons. As discussed in [12, 13], the Cartan torsion tensor plays the role of spin source in the gravitational field equations where the affine connection is not simply Levi-Civita. Furthermore, as demonstrated in [106, 117 119], torsion plays an important role in Extended Theories of Gravity since brings further gravitational degrees of freedom responsible of chiral interactions. In other words, torsion is not only the source of spin but, thanks to the non-trivial structure of connections $\Gamma_{\beta \gamma}^{\alpha}$, can give rise to chiral interactions of geometric origin [120]. This means that the $S U(2)_{L}$ and $S U(3)$ could be related to a gravitationally induced symmetry breaking process where torsion plays a fundamental role. Due to these facts, the present approach has to be generalized including torsion fields. Phenomenological studies considering torsion and fermion interactions are already reported in [121].

Furthermore, as shown in [120], space-like, time-like and null torsion tensors, generated by non-trivial combinations of vector and bi-vector fields, can be classified and represented by matrices which could explain mass matrices of fermions and the hierarchy in the generation of quarks. This approach agrees with other approaches where the effects of gluonic condensates in holographic QCD can be encoded in suitable deformations of 5D metrics (see e.g. 91]).

A detailed study in this sense will be the argument of forthcoming studies.

The validity of the presented scheme could be reasonably checked at LHC in short time, due to the increasing luminosities of the set up. In fact, the LHC experiments (in particular ATLAS and CMS) are indicating, very preliminary, the presence of resonances and condensate states that confirm the Standard Model but, up to now, cannot be considered as evidences for the Higgs boson [113]. Similar very preliminar results, but with larger integrated luminosity, are reported also by the CDF collaboration at Fermi Lab 122]. The interpretation of such data could be that the gravitational ghosts (discussed 
here) would induce the formation of resonances and condensates without the presence of any Higgs field as discussed in the previous sections.

A final remark deserves the treatment of ghost states that we have introduced here. According to the BecchiRouet-Stora (BRS) formulation of gauge theories, negative norm states or ghosts are allowed to propagate and are eliminated by applying a particular condition (BRScondition) onto the state vectors [123]. The approach can be expressed in terms of path integrals. As consequence, the vacuum is the ground state and ghosts do not generate the negative energy states without conflicting with the Copenhagen Interpretation of Quantum Mechanics. In our case, we are in a more general context. Our gravitational ghosts are related to non-local effects connected to the fact that $G L(4)$ is a non-unitary group where some sub-groups can be unitary. Such ghosts are related to the gravitationally-induced symmetry breaking process and to the further degrees of freedom emerging in the Extended Theories of Gravity. In other words, they are not the ghosts of the standard BRS mechanism but further gravitational modes related to non-locality. In a very general sense, we are compatible with the Many Worlds Interpretation of Quantum Mechanics 124 since any projection from the 5D manifold realizes a different universe and then a different effective theory of gravity. This point will be discussed in details in further works.

\section{ACKNOWLEDGMENTS}

The Authors wish to thank Sergio Bertolucci for useful comments, common discussions and suggestions which allowed to improve the paper.
[1] G. Basini and S. Capozziello, "Open Quantum Relativity", in Classical and Quantum Gravity Research, Ed. M.N. Christiansen, Nova Science Publishers, Inc., New York (2008).

[2] C. Rovelli, Quantum Gravity, Cambridge University Press, Cambridge (2004).

[3] G. Basini and S. Capozziello, Gen. Rel. Grav., 35, 2217 (2003).

[4] G. Basini and S. Capozziello, Mod. Phys. Lett. A 20, 251 (2005).

[5] G. Basini and S. Capozziello, Int. Jou. Mod. Phys. D 15, 583 (2006).

[6] R. Utiyama, Phys. Rev. 101, 1597 (1956).

[7] C. N. Yang and R. L. Mills, Phys. Rev. 96, 191 (1954).

[8] T.W. Kibble, J. Math. Phys. 2, 212 (1960).

[9] E. Cartan, Ann. Ec. Norm. 42, 17 (1925).

[10] D.W. Sciama, On the analog between charge and spin in General Relativity, in Recent Developments in General Relativity, Festschrift for Leopold Infeld, (1962) 415, Pergamon Press, New York.

[11] R. Finkelstein, Ann. Phys. 12, 200 (1961).

[12] F. W. Hehl et al., J. Math. Phys. 12,1334 (1970) .

[13] F.W. Hehl et al., Rev. Mod. Phys. 48, 393 (1976).

[14] H. I. Arcos and J. G. Pereira, Int. J. Mod.Phys. D 13, 2193 (2004).

[15] F. Mansouri et. al., Phys. Rev. D 13, 3192 (1976) .

[16] F. Mansouri, Phys. Rev. Lett. 42, 1021 (1979).

[17] G. Grignani et. al., Phys. Rev. D 45, 2719 (1992).

[18] L. N. Chang et al., Phys. Rev. D 13, 235 (1976) .

[19] F. W. Hehl and J. D. McCrea, Found. Phys. 16, 267 (1986).

[20] A. Inomata et. al., Phys. Rev. D 19 , 1665 (1978) .

[21] C. G. Callan, S. Coleman, J. Wess and B. Zumino, Phys. Rev. 117, 2247 (1969).

[22] S. Coleman, J. Wess and B. Zumino, Phys. Rev. 117, 2239 (1969).

[23] C. J. Isham, A. Salam and J. Strathdee, Ann. of Phys. 62, 98 (1971).

[24] A.B. Borisov and V.I. Ogievetskii, Theor. Mat. Fiz. 21, 329 (1974).

[25] E. A. Ivanov and V. I. Ogievetskii, Gauge theories as theories of spontaneous breakdown, Preprint of the Joint Institute of Nuclear Research, E2-9822 3-10 (1976).

[26] L. N. Chang et al., Phys. Rev. D 17, 3168 (1978) .

[27] K. S. Stelle et al., Phys. Rev. D 21, 1466 (1980).

[28] E. A. Ivanov and J. Niederle, Phys. Rev. D 25, 976 (1982).

[29] E. A. Ivanov and J. Niederle, Phys. Rev. D 25, 988 (1982).

[30] D. Ivanenko and G. A. Sardanashvily, Phys. Rep. 94 , 1 (1983).

[31] A. Lord and P. Goswami, J. Math. Phys. 27, 3051 (1986) .

[32] E. A. Lord and P. Goswami, J. Math. Phys. 29, 258 (1987)

[33] Y. Ne'eman and T. Regge, Riv. Nuovo Cimento 1 1, (1978).

[34] A. Lopez-Pinto, A. Tiemblo and R. Tresguerres, Class. Quant. Grav. 12, 1503 (1995).

[35] J. Julve et. al., Class. Quantum Grav. 12, 1327 (1995).

[36] R. Tresguerres and E. W. Mielke, Phys.Rev. D 62, 044004 (2000).

[37] R. Tresguerres, Phys. Rev. D 66, 064025 (2002).

[38] A. Tiemblo and R. Tresguerres, Eur.Phys.J. C 42, 437 (2005).

[39] A. Tiemblo and R. Tresguerres, Recent Res.Devel.Phys. 5, 1255 (2004).

[40] S. Capozziello, G. Lambiase, C. Stornaiolo, Ann. Phys. (Leipzig) 10, 713 (2001).

[41] S. Capozziello and M. De Laurentis, Int. J. Geom. Meth. Mod. Phys. 6, 1 (2009).

[42] G. Basini, S. Capozziello, and G. Longo, Phys. Lett. A 311, 465 (2003).

[43] T. Appelquist, A. Chodos, P.G.O. Freund, Modern Kaluza-Klein Thories, Frontiers in Physics, AddisonWesley Publishing Company, Inc. Redwood, CA (1987).

[44] G.G. Ross, Grand Unified Theories, Benjamin, Menlo Park, CA (1985).

[45] G. Basini, A. Morselli, M. Ricci, La Riv. del N. Cimento 12 (1989) 4.

[46] I.L. Buchbinder, S.D. Odintsov, and I.L. Shapiro, Effective Action in Quantum Gravity, IOP Publishing (1992) 
Bristol.

[47] S.Nojiri and S.D. Odintsov, Int. J. Meth. Mod. Phys. 4, 115 (2007).

[48] S. Capozziello, M. Francaviglia, Gen. Rel. Grav.40, 357 (2008).

[49] S. Capozziello and V. Faraoni, Beyond Einstein Gravity, Fundamental Theories of Physics Vol. 170, Springer Ed., Dordrecht (2011).

[50] S. Nojiri and S.D. Odintsov, arXiv:1011.0544 [gr-qc], to appear in Phys. Rep. (2011).

[51] S. Capozziello, E. Elizalde, S. Nojiri, S. D. Odintsov, Phys. Lett. B 671, 193 (2009).

[52] S. Jhingan, S. Nojiri, S.D. Odintsov, M. Sami, I. Thongkool, S. Zerbini, Phys. Lett. B 663, 424 (2008).

[53] M. Nakahara, Geometry, Topology and Physics Second Edition, Graduate Student Series in Physics, (Institute of Physics, Bristol and Philadelphia 2003)

[54] G. Giachetta, J. Math. Phys. 40, 939 (1999).

[55] R. Gilmore, Lie Groups, Lie Algebras, and Some of their Applications. Krieger Pub. Co., Malabar (1994).

[56] G. F. B. Riemann,G.F.B., Uber dieHypothesen, welche der Geometrie zu Grunde liegen, Abhand. K. Ges. Wiss. Gottingen, 13, 133 (1868); English translation by Clifford, W. K. Nature 8, 14, (1873); reprinted and edited by Weyl, H., Springer, Berlin, 1920. Included in its Gesammelte Mathematische Werke, wissenschaftlicher Nachlass und Nachtrage, eds. Weber, H., Dedekind, R., Teubner, B. G., Leipzig, (1892); 2d ed. Dover Publ., New York.

[57] R. M. Wald, General Relativity, The University of Chicago Press, (1984).

[58] B. Coll, J. Llosa and D. Soler, Gen. Rel. Grav. 34, 269 (2002).

[59] S. Weinberg, Gravitation and Cosmology, Wiley, New York (1972).

[60] J.E. Campbell A Course of Differential Geometry, Clarendon, Oxford (1926).

[61] G. Basini, S. Capozziello, Europhys. Lett. 63, 166 (2003).

[62] G. Basini, S. Capozziello, G. Longo, Astron. Nach. 324, 275 (2003).

[63] V. Faraoni, Class. Quantum Grav. 22, 32352 (2005).

[64] C. Rubano and P. Scudellaro, Gen. Relativ. Grav. 37, 521 (2005).

[65] M. Ferraris, M.Francaviglia, in: Mechanics, Analysis and Geometry: 200 Years after Lagrange; Editor: M. Francaviglia, Elsevier Science Publishers B:V:, (1991).

[66] G. Magnano and L.M. SokoLowski, Phys. Rev. D 50, 5039 (1994).

[67] A. Nunez and S. Solganik, Phys. Lett. B 608, 189 (2005).

[68] T. Chiba, JCAP 0503, 008 (2005).

[69] K. S. Stelle, Gen. Rel. Grav. 9, 353 (1978).

[70] H. van Dam and M. J. G. Veltman, Nucl. Phys. B 22, 397 (1970).

[71] C. W. Misner, K. S. Thorne and J. A. Wheeler - "Gravitation" - W.H.Feeman and Company - (1973).

[72] S. Capozziello, M. De Laurentis, C. Corda, Phys. Lett. B 699, 255 (2008).

[73] R. Emparan and J. Garriga, JHEP 0603, 028 (2006).

[74] A. Achucarro, R. Gregory and K. Kuijken, Phys. Rev. D 52, 5729 (1995).

[75] M. Maggiore, Phys. Rep. 331, 283 (2000).

[76] I. Antoniadis, S. Dimopoulos and A. Giveon, JHEP
0105, 055 (2001)

[77] C. A. Mead, Phys. Rev. 135, B849 (1964); T. Padmanabhan, Class. Quant. Grav. 4, L107 (1987); X. Calmet, M. Graesser and S. D. H. Hsu, Phys. Rev. Lett. 93, 211101 (2004); X. Calmet, M. Graesser and S. D. H. Hsu, Int. J. Mod. Phys. D 14, 2195 (2005).

[78] D. Amati, M. Ciafaloni and G. Veneziano, Phys. Lett. B 216, 41 (1989); see also D. Amati, M. Ciafaloni and G. Veneziano, Int. J. Mod. Phys. A 3, 1615 (1988).

[79] P. Meade and L. Randall, Jou. High. Energy Phys. 0805, 003, 2008)

[80] J. L. Feng and A. D. Shapere, Phys. Rev. Lett. 88, 021303 (2002)

[81] L. A. Anchordoqui, J. L. Feng, H. Goldberg and A. D. Shapere, Phys. Rev. D 65, 124027 (2002)

[82] L. A. Anchordoqui, J. L. Feng, H. Goldberg and A. D. Shapere, Phys. Rev. D 68, 104025 (2003)

[83] A. Ringwald and H. Tu, Phys. Lett. B 525, 135 (2002).

[84] M. Kowalski, A. Ringwald and H. Tu, Phys. Lett. B 529, 1 (2002)

[85] C. H. Llewellyn Smith, Phys. Lett. B 46, 233 (1973) .

[86] B. W. Lee, C. Quigg and H. B. Thacker, Phys. Rev. Lett. 38, 883 (1977).

[87] B. W. Lee, C. Quigg and H. B. Thacker, Phys. Rev. D 16, 1519 (1977).

[88] C. E. Vayonakis, Lett. Nuovo Cim. 17, 383 (1976).

[89] G. Dvali, G. F. Giudice, C. Gomez and A. Kehagias, CERN-PH-TH-226 (2010).

[90] V. Visnjic, Nuovo Cim. A 101, 385 (1989).

[91] L. Cappiello and G. D'Ambrosio, Eur. Phys. J. C 69, 315 (2010).

[92] G. 't Hooft, "Topological aspects of quantum chromodynamics", Lectures given at International School of Nuclear Physics: 20th Course: Heavy Ion Collisions from Nuclear to Quark Matter (Erice 98), Erice, Italy, 1725 Sep 1998, "Topological aspects of quantum chromodynamics," hep-th/9812204, published in "Erice 1998, From the Planck length to the Hubble radius," 216-236.

[93] G. 't Hooft, in "Recent Developments In Gauge Theories," Cargèse 1979, ed. G. 't Hooft et al. Plenum Press, New York, 1980, Lecture II, p.117, "Recent Developments In Gauge Theories. Proceedings, Nato Advanced Study Institute, Cargèse, France, August 26 - September 8, 1979."

[94] J. Hewett and T. Rizzo, JHEP 0712, 009 (2007).

[95] H. Arason, D. J. Castano, B. Keszthelyi, S. Mikaelian, E. J. Piard, P. Ramond and B. D. Wright, Phys. Rev. D 46, 3945 (1992).

[96] M. Fabbrichesi, R. Percacci, A. Tonero and O. Zanusso, Phys.Rev. D 83, 025016 (2011).

[97] R. Percacci and O. Zanusso, Phys. Rev. D 81, 065012 (2010)

[98] T. Appelquist and M. S. Chanowitz, Phys. Rev. Lett. 59, 2405 (1987) [Erratum-ibid. 60, 1589 (1988)].

[99] F. Maltoni, J. M. Niczyporuk and S. Willenbrock, Phys. Rev. Lett. 86, 212 (2001).

[100] F. Maltoni, J. M. Niczyporuk and S. Willenbrock, Phys. Rev. D 65, 033004 (2002).

[101] D. A. Dicus and H. J. He, Phys. Rev. D 71, 093009 (2005).

[102] D. A. Dicus and H. J. He, Phys. Rev. Lett. 94, 221802 (2005).

[103] S. Capozziello, J. Matsumoto, S. Nojiri, S. D. Odintsov, Phys. Lett. B 693, 198 (2010). 
[104] N.Birrell and P.C.Davies, Quantum Fields in Curved Space, Cambridge Univ. Cambridge (1984).

[105] A. Schwarz, Topology for Physicists, (Springer-Verlag: Berlin Heidelberg, 1994)

[106] S. Capozziello, R. Cianci, C. Stornaiolo, S. Vignolo, Int. J. Geom. Meth. Mod. Phys. 5, 765 (2008).

[107] S. Capozziello, R. de Ritis, A.A. Marino, Class. Quantum Grav. 14, 3243 (1997).

[108] S. Weinberg, in Understanding the Fundamental Constituents of Matter, ed. A. Zichichi (Plenum Press, New York, 1977); in General Relativity, ed. S. W. Hawking and W. Israel (Cambridge University Press, 1979): 700.

[109] S. Capozziello, M. De Laurentis, M. Francaviglia, Astrop. Phys. 29125 (2008).

[110] X. Calmet, S. D. H. Hsu, D. Reeb Phys. Rev. D 77, 125015 (2008).

[111] X. Calmet, S. D. H. Hsu, Phys. Lett. B 663, 95 (2008).

[112] A. Pich, arXiv: 0705.4264[hep-ph] (2007).

[113] G. Aad et al. (The ATLAS collaboration) Eur. Phys. J. C 71, 1512 (2011).
[114] I. Antoniadis, N. Arkani-Hamed, S. Dimopoulos and G. R. Dvali, Phys. Lett. B 436, 257 (1998).

[115] P. Minkowski, Phys. Lett. B 71, 419 (1977).

[116] A. Zee, Phys. Rev. Lett. 42, 417 (1979).

[117] S. Capozziello, R. Cianci, C. Stornaiolo and S. Vignolo, Class. Quantum Grav., 24, 6417, (2007).

[118] S. Capozziello, R. Cianci, C. Stornaiolo and S. Vignolo, Phys. Scripta, 78, 065010, (2008).

[119] S. Capozziello and S. Vignolo, Ann. Phys., 19, 238, (2010).

[120] S. Capozziello, G. Lambiase and C. Stornaiolo, Ann. Phys. 10, 713 (2001).

[121] A.S. Belyaev, I.L. Shapiro, and M.B.A. do Vale Phys. Rev. D 75, 034014 (2007).

[122] T. Aaltonen et al. arXiv: 1104.0699 [hep- ex] (2011).

[123] M. Kaku, Quantum Field Theory, Oxford Univ. Press, Oxford (1993).

[124] J. B. Hartle, Prediction In Quantum Cosmology", in Gravitation in Astrophysics" Cargese 1986, Proceedings, S. Carter, J. B. Hartle eds, 329-360 (1986). 